\title{
4. Die fünf untersuchten queeren Projekte: Eine Beschreibung
}

Queere Politiken sind im deutschsprachigen Raum aus dem US-amerikanischen auf unterschiedliche Arten übernommen worden. »Queer«, wie es sich in den USA formiert hat, steht für eine kritische Haltung gegenüber eindeutigen Identitätspolitiken, denen Bündnisse jenseits von eindeutigen Identitäten im Kampf gegen den Tod entgegengesetzt wurden. Im deutschsprachigen Raum hat es im Zuge der Aneignung des Begriffs »queer« Bedeutungsverschiebungen gegeben. Hier gilt »queer« zum Teil einfach als Überbegriff für LGBT-Politiken ${ }^{1}$ oder bezeichnet Politiken, die »inklusiver « sind. ${ }^{2}$ Sowohl für den US-amerikanischen als auch für den deutschsprachigen Raum zeigt sich, dass queere Politiken auf verschiedene Bewegungen (Schwulenbewegung, Lesbenbewegung, den Trans* - und migrantischen Aktivismus) zurückgehen, für die oft jeweils unterschiedliche Themen (z.B. strafrechtliche Verfolgung, Aids, feministische Themen, Pathologisierung, Rassismus, Antisemitismus) relevant waren und bis heute sind. Aktuelle deutschsprachige queere Aktivismen - so auch die Türkis Rosa Lila Villa, die Milchjugend, LesMigraS, TransInterQueer e.V. und das Jugendnetzwerk Lambda BB können entsprechend als so etwas wie ein Konglomerat aus all diesen Bewegungen und damit einhergehenden Debatten verstanden werden. Nachdem queere Politiken und deren Kritik an (eindeutigen) Identitätspolitiken nun bereits theoretisch und bewegungsgeschichtlich kontextualisiert wurden, soll als Nächstes der Fokus auf diejenigen queeren Projekte gelegt werden, die im Zentrum der folgenden Analyse stehen. Hier$\mathrm{zu}$ werden zu Beginn die Kriterien nach denen die fünf queeren Projekte ausgewählt wurden, beschrieben. Anschließend folgt eine detaillierte Beschreibung der einzelnen Einrichtungen.

\section{Eine Auswahl}

Ausgehend von den theoretisch dargestellten Kritiken an (eindeutigen) Identitätspolitiken und der expliziten bewegungsgeschichtlichen Verortung von »queer« als Bündnispolitiken jenseits schwul-lesbischer Identitätspolitiken, steht im Zentrum dieser Arbeit 
die Frage danach, welche Rolle Identitäten für das politische Subjekt aktueller queerer Politiken im deutschsprachigen Raum spielen. Um sich dieser Forschungsfrage zu nähern, wurde zunächst der Forschungsgegenstand - aktuelle queere Politiken - benannt und eingegrenzt. Hierzu wurde zu Beginn theoretisch und empirisch der potentielle Gegenstand gesichtet und bestimmt. Die Samplingstrategie ist durch eine abwechselnde »Offenheit « und »Theoriegeleitetheit « charakterisiert. ${ }^{3}$ Zunächst ging es darum, den Ist-Zustand der queer-politischen Landschaft im deutschsprachigen Raum zu recherchieren. Gleichzeitig wurde aber auch versucht, diesen theoretisch näher zu bestimmen. Bezug nehmend auf die soziale Bewegungsforschung kann - so erste Erkenntnisse - »queer im deutschsprachigen Raum insofern als soziale Bewegung ${ }^{4}$ verstanden werden, als es sich um oppositionelle Versuche handelt, soziale Räume neu und anders $\mathrm{zu}$ besetzen. Allerdings lassen sich soziale Bewegungen »als >Gegenstand [...] kaum isolieren «. ${ }^{5}$ Um einen konkreten Forschungsgegenstand auswählen zu können, wurde deswegen auf den Projektbegriff aus der feministischen Bewegungsforschung zurückgegriffen. Ausgehend von der Formierung verschiedener Einrichtungen in der Frauenbewegung der 1970er-Jahre, spricht die Frauenbewegungsforschung von »Frauenprojekten«. Dieser im feministischen Kontext verwendete Projektbegriff kommt den empirisch gesichteten queeren Politiken im deutschsprachigen Raum sehr nahe. Analog zur Definition von Frauenprojekten bestehen auch queere Politiken im deutschsprachigen Raum vor allem in Form von selbstorganisierten Einrichtungen, die von und für queere Menschen geschaffen wurden. ${ }^{6}$ Eine nähere Bestimmung queerer Politiken durch den Projektbegriff ermöglicht es auch, die Kriterien für die konkrete Auswahl der Projekte im Sinne einer "maximalen « und "minimalen Kontrastierung « festzulegen. ${ }^{7}$ So wurden fünf queere Projekte aus Deutschland, Österreich und der Schweiz ausgewählt, die sich in Hinsicht auf manche Bestimmungen des Projektbegriffs im Sinne der maximalen Kontrastierung unterscheiden, im Hinblick auf andere Aspekte im Sinne der minimalen Kontrastierung ähneln.

Die fünf Einrichtungen, die im Zentrum der folgenden Analyse stehen, sind die Milchjugend, die Türkis Rosa Lila Villa, das Jugendnetzwerk Lambda BB, TransInterQueer e.V. und LesMigraS. Bei allen fünf Projekten handelt es sich - analog zur Bestimmung von

3 Andreas Witzel: »Auswertung problemzentrierter Interviews. Grundlagen und Erfahrungen«, in: Rainer Strobl (Hg.), Wahre Geschichten? Zu Theorie und Praxis qualitativer Interviews, BadenBaden: Nomos-Verlag 1996, S. 49-75, hier S. 52.

4 Eine soziale Bewegung wird als ein Verbund verschiedener Gruppen, die strukturell auf einem »mittleren Organisationsgrad« organisiert sind und ein gemeinsames Ziel teilen, verstanden. Aktuelleren feministischen Bewegungsforscher*innen zufolge können diese auch als Orte, die Dissens und Konflikte in sich aufnehmen, gefasst werden. Vgl. Susanne Maurer: „Soziale Bewegung «, in: Fabian Kessl/Christian Reutlinger (Hg.), Handbuch Sozialraum, Wiesbaden: Springer Verlag 2019, S. 1-23.

5 Ebd., S. 7 f.

6 Yvonne P. Doderer/Beate Kortendiek: »Frauenprojekte: Handlungs- und Entwicklungsräume feministischer Frauenbewegungen«, in: Ruth Becker/Beate Kortendiek (Hg.), Handbuch Frauen- und Geschlechterforschung, Wiesbaden: Springer Verlag 2010, S. 887-894, hier S. 887f.

7 Oliver Dimbath/Michael Ernst-Heidenreich/Matthias Roche: »Praxis und Theorie des Theoretical Sampling. Methodologische Überlegungen zum Verfahren einer verlaufsorientierten Fallauswahl«, in: Forum Qualitative Sozialforschung 19 (2018). 
Frauenprojekten - um selbstorganisierte Einrichtungen in Vereinsform. Auch in Bezug auf die Form der Selbstorganisation sowie den Grad der Institutionalisierung und Professionalisierung ist bei der Projektauswahl nach dem Primat des minimalen Kontrasts vorgegangen worden. Die ausgewählten Projekte sind, wie es bei den meisten queeren Einrichtungen der Fall ist, juristisch als eingetragene Vereine organisiert, die sich selbst finanzieren, aber auch verschiedene Formen staatlicher und anderer Förderungen erhalten. Die Arbeit findet zumeist ehrenamtlich statt, vereinzelt gibt es aber auch bezahlte Stellen für die Koordination der Vereine, die Durchführung einzelner Projekte, ${ }^{8}$ oder auch für die Beratungen. ${ }^{9}$ Ähnlich wie Frauenprojekte sind auch queere Projekte vermehrt in Großstädten zu finden. Da nicht nur historisch gesehen, sondern auch aktuell, urbane Räume zentrale Orte queerer Kämpfe und Öffentlichkeiten sind, ${ }^{10}$ wurde die Auswahl der Projekte auf die jeweils größten Städte der ausgewählten Länder, nämlich Berlin, Wien und Zürich beschränkt. Der Fokus auf Großstädte ermöglichte es, ausreichend queere Einrichtungen zu finden, die den Auswahlkriterien entsprachen und eine Beantwortung der Forschungsfrage möglich machten. ${ }^{11}$ Auch die Definition von Frauenprojekten als Initiativen, die »sich vor dem Hintergrund der Grundsätze feministischer Theorie und Praxis situieren «, ${ }^{12}$ spielte übertragen auf queere Projekte eine Rolle. Die theoretische Ausarbeitung, die bewegungsgeschichtliche Situierung, aber auch die konkrete queere politische Praxis zeigt, dass "queer« sehr unterschiedlich gefasst wird. Gleichzeitig ist »queer « eigentlich ein Begriff, der sich gerade jeglicher Festschreibung zu entziehen versucht. Ausgehend von der Fragestellung, die wiederum vor dem Hintergrund bereits dargestellter theoretischer wie bewegungsgeschichtlicher Situierung formuliert wurde, markiert »queer als Auswahlkriterium für den Forschungsgegenstand dieser Arbeit zwei wesentliche Aspekte. Zum einen sollte eine Kritik an Heteronormativität - also geschlechter- und sexualpolitische Kämpfe im Fokus der politischen Arbeit der Einrichtungen stehen, ${ }^{13}$ zum anderen sollten die Projekte sich selbst in einem identitätskritischen Sinne als »queer« verstehen. In beiden Fällen handelt es sich um Setzungen, die aus einer theoretischen sowie bewegungsgeschichtlichen Verortung heraus stattgefunden haben beziehungsweise in ihnen begründet werden und auch anders hätten gesetzt werden können. ${ }^{14}$ Es handelt sich um Ent-

8 In dem Fall sind konkrete Projekte gemeint, die in den Einrichtungen umgesetzt werden, und nicht die Organisation, die selbst auch als ein Projekt bezeichnet wird.

9 Y. P. Doderer/B. Kortendiek: Frauenprojekte: Handlungs- und Entwicklungsräume feministischer Frauenbewegungen, S. $887 f$.

10 Natalie Oswin: »Queer Worldings in the Urban Age«, in: sublurban. zeitschrift für kritische stadtforschung 3 (2015), S. 99-110.

11 Der einseitige Fokus auf queer in urbanen Räumen in wissenschaftlichen Forschungsarbeiten reproduziere, so eine Kritik, die Unsichtbarkeit queerer Räume in ländlichen Gegenden. Vgl. Emma Vickers: »Queer Sex in the Metropolis? Place, Subjectivity and the Second World War«, in: Feminist Review 96 (2010), S. 58-73.

12 Y. P. Doderer/B. Kortendiek: Frauenprojekte: Handlungs- und Entwicklungsräume feministischer Frauenbewegungen, S. 887.

13 Insofern, als »queer ein offener Begriff ist, bezeichnen sich beispielsweise auch antirassistische oder anti-ableistische Projekte als »queer«.

14 Sicherlich ist es auch eine Entscheidung, die damit zusammenhängt, dass mein persönliches Interesse für die Forschungsfrage aus geschlechtertheoretischen wie geschlechterpolitischen Debatten 
scheidungen und damit auch um Positionierungen für ein bestimmtes Verständnis von "queer«, die nicht getroffen wurden, um »queer « festzuschreiben, ${ }^{15}$ sondern um einen Forschungsgegenstand zu bestimmen, der Erkenntnisse für die Fragestellung ${ }^{16}$ dieser Arbeit verspricht. Gleichzeitig bedeutet diese Festschreibung in Form der Benennung eines kleinsten gemeinsamen Nenners nicht, auf die Darstellung einer gewissen Vielfältigkeit queerer Einrichtungen durch die Projektauswahl zu verzichten. Denn, ana$\log \mathrm{zu}$ Frauenprojekten, arbeiten auch queere Einrichtungen $» \mathrm{zu}$ verschiedenen Themenschwerpunkten $\ll .{ }^{17}$ Im Sinne einer maximalen Kontrastierung unterscheiden sich die fünf ausgewählten Projekte in Bezug auf die Themenschwerpunkte beziehungsweise die Zielgruppe (entsprechend unterscheiden sich auch die Themenschwerpunkte). Sie arbeiten mit Jugendlichen (Milchjugend, Lambda BB), zu Trans* - und Inter*-Themen (TransInterQueer e.V.), zu Gewalt und Mehrfachdiskriminierung (LesMigraS) oder verstehen sich als Wohn- und Beratungsprojekt (Türkis Rosa Lila Villa). Ein maximaler Kontrast in Bezug auf den spezifischen Fokus der einzelnen Projekte sollte die Vielfalt der verschiedenen queeren Projekte, die im deutschsprachigen Raum existieren - soweit das auch nur annähernd möglich ist - , abbilden. ${ }^{18}$ Zudem schien die Frage, ob bestimmte Themen (z.B. Gewalt) oder die Anordnung um bestimmte Personengruppen (z.B. Jugendliche, Trans*-Personen oder Personen mit Mehrfachdiskriminierungserfahrung) eine bestimmte Art der Verhandlung des queeren politischen Subjekts mit sich bringt, Erkenntnisse zu versprechen. Des Weiteren stellen die ausgewählten queeren Projekte analog zu den Frauenprojekten einen »wesentlichen Bestandteil feministischer Öffentlichkeit « - in diesem Fall queerer Öffentlichkeit - dar. ${ }^{19}$ Die Sichtung queerer Projekte hat ergeben, dass viele Einrichtungen selbst an einer (queeren) Öffentlichkeit teilhaben, indem sie eigene Medien wie Flyer, Broschüren, Pressemitteilungen, Zeitschriften, Artikel und Redebeiträge produzieren. Sie nehmen auch an Community-Veranstaltungen, wie beispielsweise CSD-Paraden oder Stadtfesten, teil. Ausgehend vom empirischen Befund, der vielfältigen Produktion von internen Medien in vielen der gesichteten queeren Projekte, ist auch die Entscheidung für eine diskursanalytische Aufarbeitung dieses Textmaterials gefallen. Entsprechend wurde die Produktion von Text- und Bildmaterial zu einem weiteren Auswahlkriterium. Durch ihre Teilhabe an einer Öffentlichkeit jenseits der eigenen Gruppe grenzen sich die ausgewählten Projekte auch von jenen

hervorgegangen ist und diese aus diesem Grund auch weiterhin im Bereich vergeschlechtlichter Kämpfe betrachtet werden sollten.

15 Auch wenn das vielleicht die ungewollte Wirkung eines solchen Forschungsprojektes ist.

16 Auch die Fragestellung stellt in ihrer Bezugnahme auf eine bestimmte queere Bewegungsgeschichte und bestimmte queere Theorien eine Positionierung dar.

17 Y. P. Doderer/B. Kortendiek: Frauenprojekte: Handlungs- und Entwicklungsräume feministischer Frauenbewegungen, S. 887.

18 Allerdings ist es nicht das Ziel, queere Politiken in ihrer Vielfältigkeit zu repräsentieren. Diese ist bereits durch andere Auswahlkriterien (wie beispielsweise die Organisation als Verein, eine gemeinsam geteilte queere identitätskritische Haltung oder die Produktion einer Öffentlichkeit) beschränkt.

19 Y. P. Doderer/B. Kortendiek: Frauenprojekte: Handlungs- und Entwicklungsräume feministischer Frauenbewegungen, S. 887. 
Formen queerer Politiken ab, die nahezu ausschließlich in Bezug auf die eigene Gruppe agieren. Mittlerweile gibt es viele queere Orte, deren Fokus hauptsächlich auf kollektiven »Care-Praktiken « liegt. ${ }^{20}$ An diesen Orten werden Austausch- und Unterstützungsräume zu gemeinsam geteilten Themen geschaffen oder Beratungen angeboten. Dementgegen wurden Projekte ausgewählt, die explizit auch jenseits solcher kollektiven Care-Praktiken agieren. Mit dieser Entscheidung sollen kollektive Care-Praktiken nicht als unpolitisch markiert werden. Im Gegenteil, sie haben das Potential, eine andere Sozialität zu imaginieren, in der gegenseitige Fürsorge und nicht individuelle Verantwortung zentral ist. ${ }^{21}$ Allerdings bringt, wie die bisherigen theoretischen Darstellungen und bewegungsgeschichtlichen Verortungen gezeigt haben, eine Verhandlung des politischen Subjekts, die über die eigene Gruppe hinausgeht, eine Verkomplizierung in Bezug auf identitätspolitische Dilemmata mit sich. Auch in Gruppen kollektiver Care-Praktiken wird durch die Art, wie diese Care-Räume angeordnet sind, ein spezifisches Wir hergestellt. Diese Gruppen können und wollen (beispielsweise um einen Safe-Space herzustellen) aber zum einen zumeist in sich geschlossen bleiben, zum anderen werden Identitäten oft in Bezug auf die individuellen Bedürfnisse von Personen verhandelt. Daher kann Identität hier eine andere (zumeist affirmativere) Rolle einnehmen als in Gruppen, die ein Wir beispielsweise mit- und gegeneinander im Zuge einer Zusammenarbeit aushandeln. Um also auch letztere Dimension mitzuberücksichtigen, wurden Projekte ausgewählt, die über die eigene Gruppe hinausgehend agieren.

Diesen theoretisch informierten sowie von der Fragestellung geleiteten Auswahlkriterien folgte eine erste Phase der Kontaktaufnahme mit den Akteur*innen der Projekte. Alle in Frage kommenden Projekte sowie der E-Mail-Verteiler »Sündikat ${ }^{22}$ wurden in einer ersten Runde angeschrieben. Im Anschreiben wurden das Ziel und die Fragestellung des Forschungsprojektes dargelegt. Zudem wurden die Projekte gefragt, ob sie sich selbst in einem identitätskritischen Sinne als "queer « verstehen, ob ein Interview möglich ist, ${ }^{23} \mathrm{ob}$ und welche Text- und Bildmaterialien produziert werden und ob diese für den diskursanalytischen Teil der Arbeit verwendet werden dürfen. Die angeschriebenen Einrichtungen wurden außerdem gebeten, noch weitere queere Projekte in ihrer Stadt zu nennen, um auch auf (online) weniger sichtbare Einrichtungen aufmerksam zu werden. In dieser ersten Anschreiberunde mussten bereits einige Projekte als möglicher Forschungsgegenstand ausgeschlossen werden: Bei manchen Projekten kam auch nach mehreren Versuchen der Kontaktaufnahme keine Rückmeldung, andere hatten keine Ressourcen für Interviews oder produzierten kein oder nur wenig

Vgl. Francis Seeck/Sannik B. Dehler: »Trans Communities of Care - Eine kollaborative Reflektion von kollektiven trans Care-Praktiken«, in: Max N. Appenroth/María d. M. Castro Varela (Hg.), Trans \& Care. Trans Personen zwischen Selbstsorge, Fürsorge und Versorgung, Bielefeld: transcript Verlag 2019, S. 255-270; Mike Laufenberg:»Communities of Care. Queere Politiken der Reproduktion«, in: Luxemburg (2012), S. 96-101.

21 Beate Binder/Christine Bischoff/Cordula Endter/Sabine Hess/Sabine Kienitz/Sven Bergmann: Care: Praktiken und Politiken der Fürsorge. Ethnographische und geschlechtertheoretische Perspektiven, Opladen: Barbara Budrich Verlag 2019, S. 12.

23 Die diskursanalytische Aufarbeitung soll durch Interviews ergänzt werden, um das Moment der Selbstkonstitution der politischen Akteur*innen zu erfassen. 
Text- und Bildmaterial. Einige Projekte, vor allem explizite Trans* - und Inter*-Projekte, verorteten sich nicht in einem identitätskritischen Sinne als »queer «. ${ }^{24}$ In einer zweiten Anschreiberunde wurden weitere Projekte und solche, von denen bis dahin keine Rückmeldung gekommen war, angeschrieben. Von den Projekten, die nach der zweiten Runde noch die Einschlusskriterien erfüllten, wurden ausgehend von dem Ziel,

a) einen maximalen Kontrast in Bezug auf Themen und Arbeitsschwerpunkte herzustellen,

b) in jeder Stadt mindestens ein Projekt repräsentiert zu haben,

c) eine möglichst optimale Vergleichbarkeit der doch sehr verschiedenen selbstproduzierten Medien ${ }^{25}$ herzustellen,

folgende fünf Projekte ausgewählt: die Milchjugend, die Türkis Rosa Lila Villa, das Jugendnetzwerk Lambda BB, TransInterQueer e.V. und LesMigraS. ${ }^{26}$

\section{Eine Beschreibung}

Im Folgenden sollen nun die fünf ausgewählten Projekte vorgestellt werden. Die Beschreibung der Projekte basiert auf der Strukturanalyse des Text- und Bildmaterials, das in den Jahren 2010 bis 2016 von den fünf Einrichtungen veröffentlicht wurde. Das bedeutet, dass alle Flyer, Plakate, Broschüren, politischen Stellungnahmen, Pressemitteilungen, Redebeiträge, Zeitschriften, Artikel und Selbstdarstellungen auf der Website, die in diesem Zeitraum entstanden sind, gelesen, zusammengefasst und in Bezug auf bestimmte Kriterien aufgearbeitet wurden. ${ }^{27}$ Darüber hinaus wurden für die Beschreibung der Projekte auch Text- und Bildmaterialien miteinbezogen, die vor 2010 und nach 2016 - bis 2017 - publiziert wurden, sowie alles Text- und Bildmaterial aus dem Analysezeitraum, auch jenes, das nicht Teil der diskursanalytischen Aufarbeitung ist. Zusätzlich wurde Literatur über die Projekte in Form klassischer Online- und Printmedien sowie transferorientierte Artikel und wissenschaftliche Arbeiten gesichtet. Des Weiteren wurden vorab bereits Interviews mit Aktivist*innen aus den jeweiligen Einrichtungen geführt. Es war dabei nicht das Ziel der Interviews, über das Material hinausgehende Informationen zu den Projekten zu generieren. Den Äußerungen der Aktivist*innen

24 Projekte, die angeschrieben und aus den eben genannten Gründen als potentielle Forschungsgegenstände wegfielen, sind: »Xartsplitta «, »ABqueer«, »Migay«, »Planet10«, »Romanescos«, »Dubist-du, VimÖ«, »Transgender Network Switzerland«, »Cladt e.V.« und »Zwischengeschlecht.org«. Nachdem die Projekte sehr unterschiedliche Medien wie Flyer, Pressemitteilungen, Broschüren, Redebeiträge oder eigene Zeitschriften produzieren, sollte jedes Medium in mindestens zwei Projekten produziert werden, um eine Vergleichbarkeit in der Analyse zu gewährleisten. Im Nachhinein stellt sich die Relevanz dieses Auswahlkriteriums als überbewertet dar, weil diese Form der Vergleichbarkeit für die Analyse nicht notwendig war.

26 Dass drei Projekte aus Berlin und nur jeweils ein Projekt aus Wien und Zürich ausgewählt wurden, hängt zum einen damit zusammen, dass die Berliner Projekte sehr viele unterschiedliche Bewegungsmedien produzieren und so eine optimale Vergleichbarkeit gewährleistet werden konnte, zum anderen aber auch damit, dass mit TriQ und LesMigraS sehr spezifische Arbeits- und Themenschwerpunkte abgedeckt wurden, die in dieser Hinsicht eine Kontrastierung ermöglichen. Dieser erste methodische Schritt der Diskursanalyse wird ausführlich in Kapitel fünf dargestellt. 
konnten aber zum Teil Informationen über die Projekte entnommen werden, die ansonsten nicht zugänglich waren. Zu einigen Aktivist"innen aus den Projekten bestand zudem E-Mail-Kontakt, der Nachfragen, insbesondere in Bezug auf die Finanzierung der Projekte, ermöglichte. Die Tatsache, dass die meisten Informationen zu den fünf Projekten auf den von ihnen selbst verfassten Darstellungen beruhen, spiegelt sich in den Beschreibungen wider. Die Art und Weise, wie die Projekte sich selbst darstellen, schreibt sich in die folgende Beschreibung ein. Das ist zum einen aufgrund der Quellenlage unvermeidbar, zum anderen ist es sogar insofern gewollt, als die Art und Weise, wie sich die jeweiligen Projekte selbst darstellen, auch für die Analyse von Bedeutung ist. Gleichzeitig ist die folgende Beschreibung aber auch eine Darstellung, die von »außen« kommt ${ }^{28}$ und der bestimmte Schwerpunktsetzungen oder Interpretationen zugrunde liegen, die - vom Standpunkt der jeweiligen Projekte aus betrachtet - durchaus auch Fehl- oder Missinterpretationen sein können.

\subsection{Die Milchjugend}

\section{Vom Freund*innenkreis zur größten queeren Jugendorganisation der Schweiz}

Die Milchjugend ist ein queeres Jugendprojekt, das den Vereinssitz in Zürich hat, aber auch über Zürich hinaus Jugendliche aus der gesamten Schweiz zusammenbringt. Welche Bedeutung die Milch in der Selbstbezeichnung der Jugendlichen hat, lässt die Milchjugend offen. Der Jubiläumsausgabe zufolge scheint sich jedoch eine Erzählung recht dominant zu halten: So soll früher das Führen eines Haushaltsheftlis - des sogenannten »Milchbüechlis« - eine der ersten Aufgaben gewesen sein, bei der junge Menschen Verantwortung übernehmen durften. ${ }^{29}$ Die Milch steht also bei diesem Versuch, dem Namen (im Nachhinein) eine Bedeutung zuzuschreiben, für Verantwortungsübernahme. Die Milchjugend ist eine Gruppe von Jugendlichen, die insofern Verantwortung übernehmen, als sie eigene alternative Welten- auch das ist eine Bezeichnung der Milchjugend - schaffen, in denen sie sich ausprobieren und politisch engagieren können. Gegründet wurde das Projekt 2012 von einem Freund*innenkreis, der sich in der eigenen Jugend selbst solche Orte gewünscht hätte. Hinter der Gründung des Projekts stand die Idee, eine Plattform für queere Jugendliche in der Schweiz zu schaffen. Diese Idee wurde in einem ersten Schritt mit der Herausgabe einer Zeitschrift - dem »Milchbüechli« verwirklicht. Ein Mitbegründer der Milchjugend erzählt im Interview, dass sie damals mit der Herausgabe einer Zeitschrift begannen, weil es ganz einfach das war, was seinerzeit ihren Fähigkeiten entsprach. ${ }^{30}$ Das »Milchbüechli« - das mittlerweile als eine der vielen "Welten« der Milchjugend gilt - begleitet die Milchjugend also schon seit den Anfängen.

Aus der Produktion der Zeitschrift heraus sind später andere Welten entstanden. Zunächst folgte die »Milchreise«, die zu Beginn eine Redaktionsreiseveranstaltung war,

Mit »von außen kommen« ist gemeint, dass ich als Autorin nicht Teil der jeweiligen Projekte bin oder war. 
mit der Zeit jedoch für alle Jugendlichen geöffnet wurde. Auch die »Molke 7«-Party wurde anfänglich organisiert, um das »Milchbüechli« zu finanzieren. In den letzten Jahren sind viele weitere Welten entstanden, wie beispielsweise das Festival »Lila«, die »Milchbar« oder die »Jugendpride«. Ihre eigenen Welten schaffen sich die Jugendlichen auch auf Ebene der Sprache, indem sie versuchen, eigene Begrifflichkeiten zu (er-)finden. So bezeichnen sie sich beispielsweise selbst als »falschsexuell«. Diese Selbstbezeichnung wird als eine gemeinsame Haltung und weniger als eine (gemeinsame) Identität verstanden und in der Tradition des Begriffs "queer« verortet. ${ }^{31}$ Inhaltlich lässt sich die Arbeit der Milchjugend in drei Säulen strukturieren:

a) Öffentlichkeitsarbeit,

b) Freizeit, Kommunikation und Kultur,

c) strukturelle und inhaltliche Aufbauhilfe.

Formal ist die Milchjugend als Verein organisiert, der die Strukturen für die verschiedenen Welten und Aktionen der »falschsexuellen « Jugend bereitstellt. ${ }^{32}$ Der Verein hat über 300 aktive Mitglieder (Stand: November/2020) und besteht aus einem mehrköpfigen Vorstand, Präsident"innen, einer Geschäftsstelle und zahlreichen Menschen, die die Leitung der verschiedenen Welten übernehmen. In der Milchjugend sind Menschen bis zum Alter von dreißig Jahren aktiv. Mittlerweile verfügt das Projekt über eine hauptamtliche Vollzeitstelle, die unter zwei Student"innen aufgeteilt ist. Diese sitzen in einem Büro in Zürich, das gleichzeitig Vereinssitz ist. Finanziert wird die Milchjugend durch eigene Einnahmen und Fördergeber*innen. Eigene Einnahmen generieren sie durch Zeitschriften-Abonnements, ${ }^{33}$ Mitgliedsbeiträge, eigene Veranstaltungen ${ }^{34}$ und über das Konzept der »Milchkühe«. ${ }^{35}$ Darüber hinaus bekommt das Projekt Förder-

31 Milchjugend: Hauptgang, Milchbüechli 2014.

32 Milchjugend: Über die Milchjugend, https://milchjugend.ch/ueber-die-milchjugend vom 12.08. 2020.

33 Wobei der finanzielle Aufwand für das »Milchbüechli«höher ist als der Ertrag. Milchjugend: Rechnung Milchjugend 2018.

34 Manche Veranstaltungen sind ein Verlustgeschäft, wie z.B. die »Milchbar«, die »Milchreise« oder die »]ugendpride«. Andere Welten bringen eigene Einnahmen ein, wie z.B. die »Milchkultur« oder das Festival »Lila«. Ebd.

35 Mit dem Aufruf auf der Homepage bittet die »falschsexuelle« Jugend um finanzielle Unterstützung. Neben Spenden gibt es die Möglichkeiten, sich als Goldkuh, Silberkuh oder Bronzekuh zu beteiligen. Die Beiträge belaufen sich auf jährlich 500, 200 und 100 Schweizer Franken. Milchjugend: Spenden, https://milchjugend.ch/spenden vom 13.08.2020. 
mittel vom Bund, ${ }^{36}$ von Stiftungen, ${ }^{37}$ von der Community und von der Aids-Hilfe. ${ }^{38}$ Mittlerweile ist die Gemeinschaft der »falschsexuellen« Jugend mehr als nur ein kleiner Freund*innenkreis: Im Jahr 2020 sind über 300 Jugendliche in der ganzen Schweiz in der Milchjugend aktiv. Damit ist die Milchjugend, wie sie selbst auf ihrer Homepage schreibt, die größte LGBTIQ*-Organisation in der Schweiz, ${ }^{39}$ und das, obwohl die Arbeit zumeist ehrenamtlich geleistet wird und das Projekt weiterhin von Jugendlichen und jungen Erwachsenen selbstorganisiert wird.

\section{Die neun Welten der Milchjugend}

Die »falschsexuelle« Jugend ist primär um ihre neun Welten herum organisiert: das »Milchbüechli«, die »Milchreise«, die »Molke 7-Party«, die »Milchbar«, die »Milchkultur«, der »Milchball«, das »Festival Lila«, Schul- und Jugendgruppen und die »Jugendpride«. Die erste Welt der Milchjugend - das »Milchbüechli« - begleitet die Milchjugend seit der Gründung. Das »Milchbüechli« ist eine eigene Zeitschrift, in der die Jugendlichen diskutieren, sich austauschen, Forderungen formulieren und von ihren Erfahrungen und Welten berichten. Über die Zeitschrift, die an vielen verschiedenen Orten in der Schweiz ausliegt, wird die Milchjugend zudem sichtbar und mobilisiert so auch viele neue Mitglieder. ${ }^{40}$ Ausgehend vom »Milchbüechli« sind, wie bereits erwähnt, die anderen Welten der Milchjugend entstanden. Es sind all diese verschiedenen Welten, die die Milchjugend zusammenhält. Größtenteils geht es darum, möglichst niederschwellige Räume zu schaffen, in denen »falschsexuelle« Jugendliche sich treffen, austauschen, ausprobieren und engagieren können. Ziel ist es, die Jugendlichen in ihrem Selbstbewusstsein als das, was sie sind, zu bestärken, sie aber gleichzeitig auch für Geschlechterthemen und Politisches zu sensibilisieren. ${ }^{41}$ Mit der Anzahl der Mitglieder und mitarbeitenden Jugendlichen verändern und erweitern sich die Welten der Milchjugend ständig und wachsen rasant an. Zurzeit ist die Milchjugend dabei, eine eigene App zu entwickeln, also auch einen Online-Raum zu schaffen, in dem sich die Jugendlichen kennenlernen und austauschen können. ${ }^{42}$ Mittlerweile erreichen sie mit

36 Dabei zahlte der Bund im Jahr 2018 25.000 Schweizer Franken Unterstützung für die Betriebsstruktur und 15.000 Schweizer Franken für das Partizipationsprojekt »Queeres Jugendzentrum«. Milchjugend: Rechnung Milchjugend 2018.

Stiftungen finanzieren das Jugendprojekt mittlerweile mit ca. 36.000 Schweizer Franken pro Jahr. Dabei handelt es sich um Stiftungen, die Jugend-, Partizipations- oder Cesundheitsprojekte unterstützen, wie Migros-Kulturprozent, Schweizerische Cemeinnützige Cesellschaft, Temperatio, Stiftung Gesundheitsförderung Schweiz, Stiftung Gesundheitsförderung Zürich, Paul Schiller Stiftung, OAK Foundation und Mercator. Ebd.

Die Community und die Aids-Hilfe fördern die Milchjugend mit ca. 20.000 Schweizer Franken im Jahr. Ebd. Die finanziellen Unterstützungen von staatlicher Seite haben, den Angaben der Milchjugend zufolge, den Aufbau der queeren Jugendgruppe in den ersten Jahren überhaupt erst möglich gemacht. Auch die Community hat die Milchjugend von Anfang gefördert. Viele private Stiftungen waren dagegen zu Beginn noch zurückhaltend mit ihrer finanziellen Unterstützung. Milchjugend: Über die Milchjugend.

Das »Milchbüechli« wird im nächsten Kapitel ausführlicher beschrieben, da es im und für den diskursanalytischen Teil der Arbeit ausführlich aufgearbeitet wurde.

Milchjugend: Über die Milchjugend,

Milchjugend: App, https://app.milchjugend.ch/ vom 15.09.2020. 
ihren verschiedenen Welten einen großen Teil der »falschsexuellen « Jugendlichen in der Schweiz. Damit sind sie maßgeblich daran beteiligt, eine offene, politisch engagierte, junge »falschsexuelle« Jugendkultur zu etablieren.

\section{Ein aktivistisches Wochenende}

Die Milchreise - die zweite Welt der Milchjugend - war ursprünglich ein Redaktionswochenende für diejenigen Jugendlichen, die am »Milchbüechli« mitarbeiteten. Das Konzept wurde aber recht bald geändert und die Milchreise als ein gemeinsames aktivistisches Wochenende ins Leben gerufen. ${ }^{43}$ Die erste Milchreise fand 2014 - zwei Jahre nach Gründung der Milchjugend - statt. Seitdem verbringen jedes Jahr zwischen siebzig und achtzig Jugendliche gemeinsam ein Wochenende in einem Ferienhaus in der Schweiz. Die Kosten werden »jugendgerecht « gehalten und betragen 20 Schweizer Franken pro Wochenende. Meistens findet sie im September oder Oktober statt; in der Dezemberausgabe des »Milchbüechli« berichten die Teilnehmer*innen dann von ihren Erlebnissen. Anmelden dürfen sich alle Jugendlichen bis zu einem Alter von 25 Jahren. Mittlerweile sind die Milchreisen sehr beliebt und relativ früh ausgebucht. Menschen, die noch nie dabei waren, haben eher eine Chance auf einen Platz als solche, die schon häufiger auf Milchreise waren. ${ }^{44}$ Entsprechend ist die Milchreise auch zu einem Ort geworden, um neue Mitglieder zu mobilisieren. Die Milchreise ist als Raum gedacht, in dem Jugendliche sich kennenlernen und austauschen können und gleichzeitig in die aktivistische Welt eingeführt werden. So finden an den Wochenenden immer auch Workshops statt, an denen auf der einen Seite queer-aktivistisches Wissen vermittelt und auf der anderen Seite der eigene Aktivismus geplant und vorangetrieben wird. In den letzten Jahren gab es beispielsweise Workshops zum Thema »Gender«, »Falschsexualität« oder »queere Geschichte«, aber auch Zukunftsworkshops, in denen zukünftige Aktionen geplant werden, oder Schreibworkshops, um neue »Schreiberlinge« für das »Milchbüechli« zu rekrutieren. Neben solchen Workshops sind die Jugendlichen aber auch gemeinsam kreativ, basteln, zeichnen, spielen Theater oder verbringen gemeinsam Zeit in der Natur und machen Sport. Abends gibt es Drag-Veranstaltungen, es wird getanzt und geredet. ${ }^{45}$ Eine Teilnehmer* in beschreibt die Erlebnisse auf der Milchreise folgendermaßen:

»Wir haben neue Freundschaften geknüpft, uns verliebt, uns wurde der Kopf verdreht, unser Wissen erweitert. Wir können vielleicht gerade nicht darauf zurückgreifen, weil unser Puls sich normalisiert und uns der heteronormative Alltag entgegenschlägt, aber die Erlebnisse der Milchreise legen sich über das Jahr hinweg auf uns nieder wie ein warmes, schützendes Fell.« 46

Die Jugendlichen haben auf der Milchreise die Möglichkeit, sich kennenzulernen, sich gemeinsam mit verschiedensten Themen auseinanderzusetzen, sich auszutauschen, und vor allem, sich in einem sicheren Raum selbst auszuprobieren.

\footnotetext{
43 Damit wurde auf den ausschließenden Charakter eines Redaktionswochenendes reagiert.

44 Milchjugend: Milchreise, https://milchjugend.ch/milchreise vom 13.08.2020.

45 Vgl. Milchjugend: Hauptgang.

46 Milchjugend: Ausgabe 22, Milchbüechli 2017.
} 


\section{Kultur- und Austauschorte}

Neben der Milchreise sind die Molke 7-Partys und die Milchbar weitere Orte, an denen die Jugendlichen der Milchjugend sich treffen, austauschen und kennenlernen können. Die Molke 7-Partys wurden ursprünglich ins Leben gerufen, um die Kosten des »Milchbüechli« gegenzufinanzieren. Seitdem finden die Partys alle zwei Monate im »Heaven« - das ist ein Club in Zürich - statt. Die Partys sind ab einem Alter von 16 Jahren zugänglich, kosten 10 Schweizer Franken Eintritt und bringen der Milchjugend zusätzliche Einnahmen. Ein weiterer Ort der Milchjugend, an dem das Sich-Kennenlernen und SichAustauschen im Vordergrund steht, ist die Milchbar. Die Milchbar ist ein regelmäßiger Treffpunkt für »falschsexuellen « Jugendliche und ihre Freund"innen an einem öffentlichen $\mathrm{Ort}^{47}$ in verschiedenen Städten in der Schweiz. Zurzeit findet die Milchbar einmal die Woche beziehungsweise alle zwei Wochen in Luzern, Baden, Bern und Winterthur statt. ${ }^{48}$ Auch die Milchkultur ist der Versuch, Räume zu schaffen, an denen die Jugendlichen sich treffen und austauschen. Im Gegensatz zum informellen Austausch gibt es im Zuge der Milchkultur allerdings ein Abendprogramm. Das können Performanceabende, Leserunden, Themenabende oder Diskussionsrunden sein, in denen beispielsweise über »queere Kunst, Musik, Normen und falschsexuelle Welten« diskutiert wird. ${ }^{49}$

Eine größere kulturelle Veranstaltung der Milchjugend ist das »falschsexuelle« Festival Lila, das 2017 sein Debüt feierte. Das Festival findet einmal im Jahr, an einem Wochenende im Oktober, statt. Auf die Frage, warum die Milchjugend ein eigenes Festival brauche, antworteten die Akteuer*innen folgendermaßen:

»Die kurze Antwort: Weil es Spaß macht. Die lange Antwort: Queere Kultur fordert heraus, kritisiert, bestärkt und ermöglicht. Das Gestalten von eigenen queeren kulturellen Räumen und Kunstformen schafft Begegnungsorte unserer Subkultur. Musik, Tanz und Show sind unsere Art, die Welt auf den Kopf zu stellen. Geschlechtlichkeit, Jugend, Beziehungen, Körper, Identität und Freundschaft werden neu interpretiert. .150

Es geht den Jugendlichen auch hier darum, alternative Welten zu schaffen, indem sie queere kulturelle Räume herstellen. Die Bezeichnung »Lila« bezieht sich auf ein Lied, das in den 1920er-Jahren in Berlin populär war. An dieses Lied anknüpfend soll das Festival an die damalige Offenheit gegenüber "Falschsexuellen « in Berlin erinnern. ${ }^{51}$ Auf dem Festival treten verschiedene Drag- und Performance-Künstler"innen, queere Bands, DJs und andere Musiker"innen auf. ${ }^{52}$ Es gibt Yogaspecials, Workshops, Gesprächs-, Austausch- und Bastelrunden und einen Poetry Slam. Es wird selbstgemachtes veganes oder vegetarisches Essen verkauft und das Festival ist für Rollstuhlfahrer"innen zugänglich. Auch hier versucht die Milchjugend, die Teilnahmekosten für die Jugendlichen gering zu halten, um das Festival möglichst inklusiv gestalten. ${ }^{53}$ Ein wei-

\footnotetext{
47 Zumeist sind es Frauen-, Jugend- und linke Räume, in denen die Jugendlichen sich treffen.

48 Milchjugend: Milchbar, https://milchjugend.ch/milchbar vom 13.03.2020.

49 Milchjugend: Milchkultur, https://milchjugend.ch/milchkultur vom 13.08.2020.

50 Milchjugend: lila, htttps://lila-festival.ch/infos vom 13.08.2020

51 Milchjugend: Ausgabe 21, Milchbüechli 2017

52 Sokee ist eine der bekanntesten Künstler*innen, die auf dem Festival auftreten.

53 Milchjugend: Lila-Festival, https://lila-festival.ch/infos vom 13.08.2020.
} 
terer Ort der Milchjugend, an dem gleichzeitig queere Kultur kreiert und an queere Geschichte angeknüpft wird, ist der Milchball. Er findet seit 2017 einmal im Jahr im Dezember statt und geht auf die Bälle des Schwulennetzwerkes »Der Kreis « ${ }^{54}$ zurück. Die ersten Bälle fanden 1948 im Theater Neumarkt statt und wurden 1960 aufgrund eines Tanzverbotes von Männern mit Männern wieder aufgelöst. ${ }^{55}$ Die Milchjugend hat diese alte Tradition aufgegriffen und 2017 das erste Mal seit fast sechzig Jahren den Milchball am gleichen Ort wie damals veranstaltet. Auch ehemalige Mitglieder des Schwulennetzwerkes »Der Kreis« werden zu den Bällen eingeladen und nehmen auch teil. Auf dem Milchball feiern »falschsexuelle« Menschen ihren Weihnachtsball, ohne sich irgendwelchen Vorstellungen zu unterwerfen, wie sie sich zu kleiden oder mit wem sie zu tanzen haben. ${ }^{56}$

\section{Schul- und Jugendgruppen}

Auch jenseits von Kultur und Party ist die Milchjugend bemüht, regelmäßige Austauschund Unterstützungsorte in der gesamten Schweiz zu schaffen. In den Jugend- und Schulgruppen verbringen die Jugendlichen gemeinsam Zeit, kochen, spielen, veranstalten Drag-Abende und tauschen sich aus. Ähnlich wie bei anderen Veranstaltungen wird auch hier Wert darauf gelegt, vor allem die erste Teilnahme an dem Treffen möglichst niederschwellig zu gestalten, indem den Jugendlichen angeboten wird, gemeinsam zum ersten Treffen zu gehen. ${ }^{57}$ Die Milchjugend ermutigt schwule, lesbische, Bi-, Pan-, Trans-, Non-binary- und queere Menschen, die in ihrer Umgebung keine Möglichkeiten zum gemeinsamen Austausch haben, selbst Gruppen zu gründen. Das Projekt versucht zudem, die Infrastrukturen für die Gründung neuer »Schul- oder Jugendgruppen« bereitzustellen. Dabei gibt es in der Milchjugend Ansprechpartner*innen, die Jugendliche konkret dabei unterstützen, eine eigene Gruppe aufzubauen.

Nachdem die Schule ein Ort ist, an dem Jugendliche viel Zeit verbringen, sollen dort Räume geschaffen werden, die den Schulalltag offener gestalten: »Ziel aller Schulgruppen ist es, dass queere Jugendliche an der Schule einen Ort haben, wo sie der großartig bunten, fabulösen Queer-Kultur frönen können und sich gegenseitig unterstützen, damit der Schulalltag ein bisschen offener wird. ${ }^{58}$ Mittlerweile hat sich aus der Milchjugend heraus bereits eine Schulgruppe am »Berufs- und Weiterbildungszentrum Brugg«, eine Gruppe im »Realgymnasium Rämibühl« und eine an der »Kantonsschule Baden« formiert. Jugendgruppen wurden in Schaffhausen, in der Schwyz und in Graubünden gegründet. Hinter den Jugendgruppen steht die Idee, dass auch Jugendliche, die nicht in den großen Städten leben, Möglichkeiten haben, sich jenseits des Internets direkt miteinander auszutauschen:

»Der Kreis« war eine an Homosexuelle gerichtete Zeitschrift, die zwischen 1943 und 1967 in Zürich erschienen ist und internationale Reichweite hatte. Auch der um die Zeitschrift organisierte Verein und dessen Feste waren europaweit bekannt. Vgl. B. Cerber: Lila ist die Farbe des Regenbogens, Schwestern, die Farbe der Befreiung ist rot: die homosexuellen Arbeitsgruppen in der Schweiz (HACH) von 1974-1995. 
»Alle, die in einer Provinzstadt oder auf dem Land aufgewachsen sind, kennen es: Mensch würde gerne mit anderen falschsexuellen Jugendlichen in Kontakt treten, doch die nächste Jugendgruppe ist in der nächsten großen Stadt und damit viel zu weit weg. Das Internet ist zwar toll und verbindet Menschen miteinander, aber eine Begegnung face to face ist immer noch etwas anderes, als elektronischer Kontakt. «"

Die Entstehung der Jugendgruppe »Whatever« in Graubünden geht auf eine besondere Geschichte zurück. Im September 2015 fand ein »Kiss-in«-Protest gegen die homophoben Aussagen eines Bischofs aus Chur in Graubünden statt. Im Zuge dieses Protestes wurde der Gründer der späteren Jugendgruppe »Whatever« mehrfach darauf angesprochen, ob es denn in Graubünden eine Gruppe für "falschsexuelle« Jugendliche gebe. Diese Nachfragen nahm er zum Anlass, eine Jugendgruppe zu gründen, die sich seitdem jeden ersten Donnerstag im Monat in den Räumen der Jugendarbeit Chur trifft.

\section{Auf der Pride}

Die Jugendpride ist ebenfalls ein Ort, an dem die Milchjugend zusammenkommt, sich austauscht, sich aber auch aktiv einbringt. Die Milchjugend ist auf jeder Pride in der Schweiz vertreten. Sie haben einen eigenen Stand auf dem Festivalgelände, an dem sie auf ihre Arbeit aufmerksam machen und um den herum sich ihr Beisammensein auf der Pride organisiert. Viele Jugendliche kommen auf der Pride zum ersten Mal mit der Community und dem Aktivismus in Kontakt. Die Jugendpride ist für die in der ganzen Schweiz verteilten Jugendgruppen aber auch eine Möglichkeit, zusammenzukommen und sich auszutauschen. "Selbstbewusst « und »gemeinsam falsch « wird auf der Pride demonstriert und gefeiert. Die Jugendlichen bespielen mit ihren Reden, Performances und Slams die etwas kleinere Politbühne und haben einen eigenen Wagen, mit dem sie auf die Parade gehen. Sie verteilen das "Milchbüechli« in der Zuschauer*innenmenge und erscheinen im Sinne ihres eigenen Pride-Mottos - »Schulter an Schulter, Hand in Hand gegen Normen - Widerstand « - gemeinsam auf der Straße. ${ }^{60}$

\subsection{Die Türkis Rosa Lila Villa}

Die Türkis Rosa Lila Villa ist Teil einer queeren Stadtgeschichte Wiens, deren Arbeit und Politik sich in den letzten Jahren zwar gewandelt hat, dessen Strukturen aber noch immer auf die Anfänge der Hausbesetzer*innenbewegung und des schwul-lesbischen Aktivismus zurückgehen. Die Villa ist heute wie damals Wohnraum, Beratungsstelle und Café-Betrieb und stellt Räumlichkeiten für externe Gruppen zur Verfügung. Dabei sind es tatsächlich die vielen, mitunter sehr unterschiedlichen Gruppen und Vereine, die sich in den Räumen der Villa treffen, die die Villa ausmachen.

59 Milchjugend: Ausgabe 19, Milchbüechli 2017.

60 Die Beschreibungen zur Jugendpride beruhen größtenteils auf eigenen Beobachtungen auf der Pride 2016. 


\section{Die Entstehungsgeschichte der Villa}

Die Türkis Rosa Lila Villa befindet sich in der Linke Wienzeile 102 in Wien und ist 1982 als der erste Ort schwul-lesbischer Zusammenarbeit aus der Hausbesetzer*innenbewegung hervorgegangen. Die Hausbesetzer*innenbewegung war zu dieser Zeit in Wien sehr aktiv. Es wurden Abbruchhäuser besetzt und als Räume für Kunst, Kultur und Politik, aber auch ganz einfach als Wohnraum eingefordert. Auch der schwul-lesbische Aktivismus in Wien begann sich in den Anfängen der 1980er-Jahre zu formieren und Aufmerksamkeit einzufordern. ${ }^{61}$ Eine Art der Stadt Wien, auf die Hausbesetzer*innenbewegung zu antworten, war das "Sonderprojekt Bulldog«. Dieses Projekt hatte das Ziel, in allen 23 Wiener Bezirken den Hausbesetzer"innen jeweils zwei Abbruchhäuser zur Verfügung zu stellen. ${ }^{62}$ Die Villa war eines dieser Häuser, das zu dieser Zeit besetzt wurde. Die meisten Hausbesetzer*innen hatten damals wenig Interesse an der Villa, weil ihnen das Haus in der linken Wienzeile zu laut und zu öffentlich war. Aber gerade diese Öffentlichkeit machte die Villa zu einem begehrten Objekt für die schwullesbischen Aktivist*innen innerhalb der Hausbesetzer*innenszene. Ihnen wurde 1982 das Haus nach zähen Verhandlungen mit der Stadt Wien unter der Bedingung zugesichert, es selbst vollständig zu sanieren und dort eine Beratungsstelle für Schwule und Lesben zu errichten. Damit verfolgte die Stadt Wien in einer Zeit, in der der Paragraph $221^{63}$ es Homosexuellen untersagte, Vereine zu gründen, und der Paragraph $220^{64}$ es unter Strafe stellte, Werbung für Homosexualität zu machen, eine vergleichsweise progressive Politik. ${ }^{65}$ Der ursprüngliche Mietvertrag galt zunächst für dreißig Jahre, er ist mittlerweile bis zum Jahr 2045 verlängert worden. Seit Beginn bekommt die Villa Subventionen von der Stadt Wien. Darüber hinaus finanziert sich die Türkis Rosa Lila Villa über Fördergelder und Mieteinnahmen. Für das Haus zahlt die Türkis Rosa Lila Villa ca. 5000 Euro Miete pro Jahr, zugleich erhält sie vom Café, das im unteren Teil des Hauses angesiedelt ist, ca. 1200 Euro Miete im Monat. Auch die Bewohner"innen und einige Projekte, die die Villa nutzen, zahlen - je nach verfügbarem Budget - eine geringe Mie-

61 M. Huber: 25 Jahre andersrum.

62 Dem »Sonderprojekt Bulldog« wird unter anderem von der Hausbesetzer*innenbewegung politisches Kalkül zugeschrieben: Zum einen soll es eine Strategie gewesen sein, die Bewegungen einzudämmen, indem die Menschen mit Abbruchhäusern zufriedengestellt wurden, zum anderen bestand die Hoffnung, durch die Anwesenheit der jungen linken Hausbesetzer*innen die Altmieter*innen aus den sanierungsbedürftigen Häusern zu drängen.

$63 \S 221$. Wer eine Verbindung einer größeren Zahl von Personen gründet, deren wenn auch nicht ausschließlicher Zweck es ist, gleichgeschlechtliche Unzucht zu begünstigen, und die geeignet ist, öffentliches Ärgernis zu erregen, ferner, wer einer solchen Verbindung als Mitglied angehört oder für sie Mitglieder wirbt, ist mit Freiheitsstrafe bis zu sechs Monaten oder mit Geldstrafe bis zu 360 Tagessätzen zu bestrafen.

$64 \S 220$. Wer in einem Druckwerk, in einem Laufbild oder sonst öffentlich zur gleichgeschlechtlichen Unzucht oder zur Unzucht mit Tieren auffordert oder sie in einer Art gutheißt, die geeignet ist, solche Unzuchtshandlungen nahezulegen, ist, sofern er nicht als an der Unzuchtshandlung Beteiligter ( $\$ 12)$ mit strengerer Strafe bedroht ist, mit Freiheitsstrafe bis zu sechs Monaten oder mit Geldstrafe bis zu 360 Tagessätzen zu bestrafen.

65 Linda Jannach: Die Entstehungsgeschichte(n) des schwul-lesbischen Hausprojektes Rosa Lila Villa in Wien. Räumliche Aneignungspraktiken und Widerstand. Masterarbeit 2015. 
te. ${ }^{66}$ Des Weiteren ist die Beratungs- und Bildungsarbeit der Beratungseinrichtungen, die »Tipps« genannt werden, finanziell gefördert. ${ }^{67}$ Die Türkis Rosa Lila Villa ist bis heute ein wichtiger Raum und Ausgangspunkt für den LGBTQ ${ }^{68}$-Aktivismus in Wien. Sie basiert auf den Säulen

a) Wohnen,

b) Beratung,

c) Café,

d) Verfügbarmachung von Räumlichkeiten für externe Projekte und politische Organisationen.

\section{Die Villa als Wohnraum}

Zu Beginn gaben viele Aktivist"innen ihre eigenen Wohnungen auf, um die Villa zu sanieren und rund um die Uhr für Beratungen zur Verfügung zu stehen. In dieser Zeit wurden zudem Notfallschlafplätze, vor allem für männliche Sexarbeiter* sowie für Gäste und Tourist*innen, in der Villa angeboten. Im Obergeschoss wurden recht bald Wohnungen für die Aktivist*innen der Villa eingerichtet. Es entstand eine Wohngemeinschaft für Lesben und eine Wohngemeinschaft für Schwule. ${ }^{69}$ Nachdem zu Beginn der Aktivismus der Hauptgrund für den Einzug in die Villa darstellte, ging es später darum, denjenigen Menschen Wohnraum zur Verfügung zu stellen, die dringenden Bedarf hatten - »etwa wegen Problemen mit der Familie.$^{70}$ Bis heute ist das kostengünstige Wohnen in den WGs ein zentraler Aspekt der Villa. Allerdings sind die WGs heute nicht mehr nur für Schwule und Lesben, sondern auch für queere, bisexuelle, Trans* - und intergeschlechtliche Menschen offen. Der "Türkis Rosa Lila Wohn*Satellit« - so werden die Wohngemeinschaften, die auch als eigener Verein eingetragen sind, bezeichnet versteht sich als Ort, der nicht nur kostengünstiges Wohnen möglich macht, sondern an dem ein Zusammenleben jenseits "heteronormativer Lebenskonzepte« stattfindet: »Wohnungstüren sind offen und es gibt auch gemeinsam genutzten Wohnraum. [...] Konzepte wie >mein Haus, mein Hund, mein Auto, meine Kinder< [...] können uns gestohlen bleiben. ${ }^{71}$ Mittlerweile spielt das Thema Wohnen nicht mehr nur in Bezug auf die WGs eine wichtige Rolle in der Villa-Politik, sondern auch in Bezug auf Geflüchtete.

66 Diese Informationen stammen von meinem Interviewpartner aus dem Jahr 2017.

67 Durch die MA13-Förderung bekommt die Villa ca. 18.000 Euro jährlich. Das ist eine Förderung für Erwachsenenbildung, außerschulische Kinder und Jugendarbeit der Stadt Wien.

Trans*-Personen wurden erst Mitte der 1990er-Jahre, vor allem im Zuge der Gründung des Vereins »TransX«, der die Räume der Villa nutzte, zunehmend Teil der Villa-Politik. Marty Huber: 35 JAHRE ROSA WIRBEL. Vom 1. Wiener Schwulen- und Lesbenhaus in die queere Intersektionalität. Ein langer Weg. IC Kultur Wien 2017, https://www.igkulturwien.net/preis-der-freien-szene/preis17 /katalogtexte/marty-huber-35-jahre-rosa-wirbel-vom-1-wiener-schwulen-und-lesbenhaus-in-die-q ueere-intersektionalitaet-ein-langer-weg/ vom 14.08.2020.

69 L.Jannach: Die Entstehungsgeschichte(n) des schwul lesbischen Hausprojektes Rosa Lila Villa in Wien, S. 8off.

70 Türkis Rosa Lila Villa : 20 Jahre Rosa Lila Villa 2002, S. 7.

71 Türkis Rosa Lila Villa : 30 Jahre Rosa Lila Villa 2012. 
Mit der Gründung von »Queer Base« in den Räumen der Villa geht es seit 2014 verstärkt auch darum, Wohnräume für geflüchtete queere Menschen zu organisieren. Es werden günstige Wohnungen angemietet und auch darüber hinaus geflüchtete queere Menschen auf der Suche nach finanzierbarem und sicherem Wohnraum unterstützt. ${ }^{72}$ Anknüpfend an die Hausbesetzer*innenbewegung bleibt so die Frage nach günstigem Wohnraum für queere Menschen bis heute ein wichtiges Thema und Anliegen der Villa. ${ }^{73}$

\section{Ein Beratungs- und Bildungsraum: Die "Tipps"}

Beratung ist von Anfang an ein wichtiger Bestandteil der Villa, nicht zuletzt auch, weil sie eine Bedingung des Pachtvertrages darstellt. Anfangs gehörte sie zu den wenigen Anlaufstellen in Wien, in der es für Schwule und Lesben, Sexarbeiter*innen und andere möglich war, sich Unterstützung in einem nicht-stigmatisierenden Umfeld zu suchen. ${ }^{74} \mathrm{Zu}$ Beginn gab es mit dem »Rosa Lila Tipp« noch eine gemeinsame Beratungsstelle für Schwule und Lesben. 1997 wurden, eigenen Angaben zufolge, die Beratungsstellen »spezifiziert«, indem jeweils »eine eigene Anlaufstelle für Lesben und Schwule» geschaffen wurde. ${ }^{75}$ Dies sollte eine optimalere Klient*innenbetreuung möglich machen. Der Trennung der Beratungen sind allerdings Konflikte zwischen den schwulen und den lesbischen Aktivist"innen vorausgegangen. ${ }^{76}$ Bis heute existieren zwei Beratungseinrichtungen: der »Lila Tipp« und der »Türkis Rosa Tipp«. Letzter hieß zunächst »Rosa Tipp«, bis in Kooperation mit dem Verein »TransX « das Türkis, das für Trans* steht, ergänzt wurde. Die »Tipps« sind ein anonymer und kostenloser Beratungsraum, in dem persönliche, telefonische und mittlerweile auch E-Mail-Beratungen angeboten werden. Es gibt festgesetzte Beratungszeiten - ein- bis zweimal pro Woche -, die auf der Homepage zu finden sind. ${ }^{77}$ Zunächst waren beide »Tipps« vor allem um die Coming-out-Beratung - der »Rosa Tipp« auch um Safer-Sex-Beratungen - herum angeordnet. Mit der Zeit erweiterte sich das Beratungsspektrum um Themen wie Fragen zu Geschlechtsidentität, Diskriminierung, Normvorstellungen, Gewalt, aber auch um rechtliche Aspekte. In ihrer Eigendarstellung betonen die Beratungen ihre feministische, queere, antihierarchische und demokratische Ausrichtung. Beide »Tipps « verfügen auch über umfangreiche Bibliotheken und ein eigenes Archiv. ${ }^{78}$ Zum Teil werden auch über die Beratungen hinaus Freizeitgruppen oder Veranstaltungen organisiert. ${ }^{79}$

Auch die Bildungsarbeit wird größtenteils von den »Tipps« organisiert. Sie hat über die Möglichkeit, eine Exkursion durch die Villa zu machen, ihren Anfang genommen.

Türkis Rosa Lila Villa : Wohnsatellit, https://dievilla.at/wohnsatellit/ vom 20.08.2020.

Angesichts der ständig steigenden Mietpreise stellt sich diese Frage vielleicht sogar mehr denn je.

L. Jannach: Die Entstehungsgeschichte(n) des schwul lesbischen Hausprojektes Rosa Lila Villa in Wien, S. 83.

Türkis Rosa Lila Villa : 20 Jahre Rosa Lila Villa, S. 7.

U. Repnik: Die Geschichte der Lesben- und Schwulenbewegung in Österreich, S. 143.

ürkis Rosa Lila Villa: Lila Tipp, https://dievilla.at/lila-tipp/ vom 20.08.2020; Türkis Rosa Lila Villa: Türkis Rosa Tipp, https://dievilla.at/traschq/ vom 20.08.2020.

Türkis Rosa Lila Villa : Bibliothek, https://dievilla.at/bibliothek/ vom 20.08.2020.

Türkis Rosa Lila Villa : Lila Tipp, https ://dievilla.at/lila tipp/ vom 20.08.2020. 
Das Angebot war und ist noch immer für Schul- und Jugendgruppen, Student"innengruppen sowie Multiplikator*innen gedacht. Die Idee ist, die Menschen durch die Villa $\mathrm{zu}$ führen und mit ihnen über verschiedenste Themen zu sprechen. Im Zuge der Exkursion werden Fragen zu Homosexualität, Gendernormen und lesbisch-schwulen Lebensrealitäten gemeinsam diskutiert und die Teilnehmer*innen bekommen eine Einführung in lesbische, schwule, Trans* - und queere Kulturen, Politiken und Geschichten sowie Informationen zur aktuellen rechtlichen Lage. ${ }^{80}$ Ziel ist es, den Besucher*innen einen direkten Einblick in einen Community-Raum zu geben und sie für Geschlechterthemen und Diskriminierung zu sensibilisieren. Im Café - der dritten Säule der Villa ist auch jenseits von Beratung und Bildung ein Einblick in die Villa-Atmosphäre möglich.

\section{Das Community-Café}

Mit den Anfängen der Villa wurde auch ein Vereinslokal mit dem Namen »Warmes Nest « eröffnet. $\mathrm{Zu}$ Beginn war das »Warme Nest« eine nicht-konsumpflichtige Teestube im Keller des Hauses. Die Idee des Cafés war, einen öffentlichen und informellen Raum des Austausches zu schaffen. ${ }^{81} 1988$ entstand dann das "Café Willendorf ${ }^{82}$ als Nachfolge zum »Warmen Nest", das im Gegensatz zu diesem ein kommerzialisierter Barund Cafébetrieb war. Nachdem das Café für einige Zeit geschlossen war, wurde es im Juni 2019 unter neuem Namen - „Villa Vida ${ }^{83}$ - und mit neuen Besitzer*innen wieder geöffnet. Das Café selbst versteht sich als Treffpunkt für Lesben, Schwule, Transgender und Freund*innen, später auch für queere Menschen. Die Aufgabe des Cafés ist es, ein öffentlicher Raum zu sein, in dem Menschen sich treffen und austauschen können. Es wird sowohl räumlich als auch in Bezug auf die Besucher*innen als das verlängerte Wohnzimmer der Villa beschrieben: »Ein eigenartiges Lokal - es gewinnt durch seine Widersprüchlichkeit. Öffentlicher Raum und verlängertes Wohnzimmer. « ${ }^{84}$ Besonders hervorgehoben wird die vielfältige frische, später auch vegane, vegetarische Küche und der Gastgarten. Der konsumpflichtige Raum des Cafés ist aber nicht der einzige Ort, an dem Menschen sich jenseits von Beratungs-, Wohn- und Bildungsangebot austauschen und treffen können. Die Türkis Rosa Lila Villa stellt immer auch Räumlichkeiten für verschiedene Gruppen und Stammtische zur Verfügung.

\section{Die Villa: Raum für politischen Aktivismus}

Neben den drei Säulen Wohnen, Beratung und Café ist die Villa auch einfach ein Raum, der von vielen verschiedenen Gruppen aus der Community genutzt wird. So sind viele

80 Türkis Rosa Lila Villa : 15 Jahre Rosa Lila Villa 2007.

81 L. Jannach: Die Entstehungsgeschichte(n) des schwul lesbischen Hausprojektes Rosa Lila Villa in Wien, S. 84.

82 Der Name »Willendorf« nimmt Bezug auf eine Venusfigur, die im Naturhistorischen Museum Wien ausgestellt ist. Die Villa versteht die Venus von Willendorf weniger als Symbol für Fruchtbarkeit, sondern als matriarchale Erdgöttin: »Die Überwindung patriarchaler Strukturen« wird in diesem Zusammenhang als einer der »wenigen Berührungspunkte zwischen Lesben und Schwulen« beschrieben. Türkis Rosa Lila Villa : 20 Jahre Rosa Lila Villa, S. 21. 
wichtige queere und LGBT-Organisationen in den Villa-Räumen entstanden und arbeiten zum Teil bis heute unter dem Dach der Villa. Der Verein »TransX« greift zum Beispiel schon seit Mitte der 9oer-Jahre auf die Räume der Villa zurück. »TransX« hat auch maßgeblich dazu beigetragen, dass die Villa sich für Trans*-Personen geöffnet hat. Damit ist die Villa nicht nur eine Einrichtung, die Räume zur Verfügung stellt, sondern die auch von der Vielfältigkeit der verschiedenen Gruppen und Vereine, die sich dort versammeln, lernt und profitiert. Eine weitere, mittlerweile feste Größe in der Villa ist das »FreiRäumchen«, das damals vom »Türkis Rosa Tipp« als fußballfreie Alternativveranstaltung zur Männer-Fußball-EM 2008 initiiert wurde. Seitdem findet das »FreiRäumchen« einmal pro Woche jeden Donnerstagabend im ersten Stock der Villa statt. Es ist ein »Treffpunk für Menschen jeden Alters, jeder Herkunft und jeglichen Geschlechts «. ${ }^{85}$ Es gibt Filmabende, Diskussionen, Partys oder Möglichkeiten des Austauschs. Zurzeit gilt das FreiRäumchen als der »transkulturellste« Treffpunkt der Stadt Wien, was unter anderem auch der Arbeit von "Queer Base« zu verdanken ist. ${ }^{86}$ Der Verein »Queer Base« wurde 2014 in den Räumen der Villa gegründet und hat es zum Ziel, geflüchtete LGBTIQ-Menschen in Österreich zu unterstützen. ${ }^{87}$ Neben Vereinen gibt es auch verschiedene Gruppen in der Villa. Es treffen sich beispielsweise eine queere und Trans*-Jugendgruppe, eine Gruppe für junge Lesben, Bisexuelle und Trans*-Personen zwischen 12 und 25 Jahren, eine Wechselgruppe, die Anonymen Alkoholiker, es gibt einen Inter*-Stammtisch, eine Gruppe, die sich mit Homosexualität und Glauben auseinandersetzt, und eine Yogagruppe. ${ }^{88}$ Aber nicht nur das, was in den Räumen der Villa ermöglicht wird, sondern auch die Villa als Raum mitten in der Wiener Innenstadt ist Teil der Villa-Politik.

\section{Die Fassade: Weil draufsteht, was auch drin ist!}

Die schwul-lesbischen Aktivist*innen der Hausbesetzer*innenbewegung waren damals unter anderem auch aufgrund der öffentlichen Sichtbarkeit an der Villa als Haus interessiert. "Weil draufsteht, was auch drin ist! - so wurde damals die Politik rund um die bunte Bemalung der Fassade der Rosa Lila Villa bezeichnet. Bis heute gilt die bunte Hausfassade der Villa als eine provokante Intervention in das Wiener Stadtbild. Für die Besucher*innen im Coming-out-Prozess bedeutet sie auch - ein »Coming Out through Coming In ${ }^{89}$ Wenn in Broschüren, Zeitungsartikeln oder auf der Homepage der Villa die Geschichte des queeren Projektes erzählt wird, ist dort fast immer auch ein Foto von der Fassade der Villa zu finden. Auf älteren Fotos ist zunächst noch die alte, abgebröckelte braune Farbe des Hauses zu sehen. ${ }^{90}$ Nur die Tür und die beiden Fenster rechts davon sind bereits rosa angestrichen. Später bekam die Fassade dann zunächst

85 Türkis Rosa Lila Villa: Freiräumchen, https://dievilla.at/freiraeumchen/vom 20.08.2020.

86 M. Huber: 35 JAHRE ROSA WIRBEL.

87 Türkis Rosa Lila Villa: Asyl, https://dievilla.at/asyl/ vom 20.08.2020.

88 Türkis Rosa Lila Villa: Beratungen und Gruppen, https://dievilla.at/beratung-und-gruppen/ vom 20.08.2020. 
die Aufschrift »1. Wiener Schwulen- und Lesbenhaus«, die dann in »Lesben- und Schwulenhaus « umgeschrieben wurde. ${ }^{91}$ Das gesamte Haus wurde lila und rosa angestrichen. Bezug nehmend auf das Motto, dass draufsteht, was drin ist, war die Gestaltung der Villa-Fassade in den letzten Jahren Grund zu Diskussionen. Sowohl das Rosa und Lila als auch die Aufschrift Lesben- und Schwulenhaus schienen nicht mehr geeignet, die Vielfalt der Personen, die in der Villa vertreten sind, zu repräsentieren. Gleichzeitig gab es Stimmen, die die historische Fassade, in die sich die Geschichte der Villa eingeschrieben hatte, nicht aufgeben wollten. Ein Kompromiss scheiterte zunächst am Budget. ${ }^{92}$ Mittlerweile haben zwei Künstler*innen die Fassade ehrenamtlich neu gestaltet. Die Tür ist türkis, und zusätzlich zum Rosa und Lila sind verschiedene surreale Figuren an die Wand gemalt, die eine vergeschlechtlichte Einordnung nicht zulassen, allerdings People of Color repräsentieren. Die Öffentlichkeit des Hauses bedeutet für die Villa aber nicht nur Sichtbarkeit, sondern setzt sie auch der Gefahr homophober, transphober und rassistischer Angriffe aus, die sich zum Teil ebenfalls in die Fassade einschreiben. ${ }^{93}$ Die Villa-Aktivist*innen nutzen die Öffentlichkeit der Hauswand aber auch selbst, um politisch zu intervenieren. So hängen sie häufig Banner vor die Wand der Villa, deren Aufdrucke und Aufschriften beispielsweise aktuelle österreichische Politiken kritisieren oder zu Solidarität in der Community aufrufen.

\subsection{LesMigraS}

LesMigraS ist ein Projekt, das schon seit den 1990er-Jahren intersektional arbeitet und aus dieser Perspektive heraus auf verschiedensten Ebenen interveniert. So gehört LesMigraS zu den wenigen Projekten mit Fokus auf der Verwobenheit von Rassismus, Sexismus, Homophobie und Transphobie im deutschsprachigen Raum. Im Zuge der Beratungs- und Empowerment-Arbeit steht vor allem die Stärkung von Menschen, die von Gewalt und Mehrfachdiskriminierung betroffen sind im Vordergrund. Entsprechend liegt der Fokus dieser Arbeit auf Sensibilisierung und Aufklärung, sowie der Entwicklung von Handlungsstrategien.

\section{Fokus Gewalt und Mehrfachdiskriminierung}

In der »Berliner Lesbenberatung « entwickelte sich seit dem Jahr 1992 ein Arbeitsschwerpunkt mit dem Fokus auf Gewalt gegen Lesben. ${ }^{94}$ Während zu Beginn Mehrfachdiskriminierung als spezifische Gewalterfahrung lesbischer Frauen im Fokus stand, rückte im Jahr 1999, mit der Gründung von LesMigraS als eigenen Arbeitsbereich, der Blick vermehrt auf weitere Aspekte der Mehrfachdiskriminierung. Der Name LesMigraS war

\footnotetext{
91 M. Huber: 25 Jahre andersrum.

92 Letztendlich wurde stattdessen das Budget für die Barrierefreiheit der Villa genutzt.

93 Türkis Rosa Lila Villa: Schatzi, wir lassen uns nicht einschüchtern!, https://dievilla.at/blog/schatziwir-lassen-uns-nicht-einschuchtern/ vom 20.08.2020.

94 LesMigraS: Chronologie der Antidiskriminierungs- und Antigewaltarbeit der Lesbenberatung, htt ps://lesmigras.de/25-jahre-antidiskriminierungsantigewaltarbeit.html vom 15.09.2020.
} 
ursprünglich eine Abkürzung für »Lesbische Migrant"innen und Schwarze Lesben«. ${ }^{95}$ Später wurde der Arbeitsbereich auf »lesbische bisexuelle Migrant"innen, Schwarze Lesben und Trans* " erweitert. Seit 2008 gilt LesMigraS als Antidiskriminierungs- und Antigewaltbereich der »Lesbenberatung Berlin e.V. " ${ }^{96}$ Zurzeit arbeiten bei LesMigraS zumeist vier bis fünf angestellte Mitarbeiter*innen, die sich selbst als interkulturelles Team beschreiben ${ }^{97}$ Neben Spenden wird LesMigraS unter dem Dach der "Lesbenberatung Berlin e.V.« vom Berliner Senat und von Stiftungen gefördert. ${ }^{98}$ Einzelne Projekte und einzelne Broschüren erhalten gesonderte Förderungen von Stadt, Land, Bund, EU oder von Stiftungen. Die Arbeit gegen Gewalt und Mehrfachdiskriminierung beruht vor allem auf den Säulen
a) Beratung,
b) Empowerment,
c) interkulturelle Öffentlichkeits- und Bildungsarbeit.

Die einzelnen Bereiche sind allerdings nicht klar voneinander trennbar, sondern überschneiden sich.

\section{Beratung zum Thema Gewalt und Mehrfachdiskriminierung}

Die Beratung von LesMigraS findet in verschiedenen Sprachen, kostenlos und anonym statt. ${ }^{99}$ Menschen können sich telefonisch, per E-Mail, im Chat oder persönlich beraten lassen. Es wird Beratung zu den Themen Gewalt (allgemein), Diskriminierung, Gewalt in Beziehungen, Empowerment, Rechtsberatung, Beratung für Inter*-Personen und Beratung zu Polizeigewalt angeboten. Ziel der Beratung ist es, gemeinsam Strategien zu finden und selbstbestimmt mit der erfahrenen Gewalt umzugehen. Eine Beratungssitzung kann bis zu 50 Minuten dauern und höchstens zehnmal in Anspruch genommen werden. Nicht nur von Gewalt oder Diskriminierung Betroffene können die Beratung von LesMigraS nutzen, sondern auch Unterstützer*innen oder Menschen, die selbst in Beziehungen Gewalt ausüben. Im Fokus der Gewalt- und Diskriminierungsberatung stehen dabei immer die Bedürfnisse der von Gewalt betroffenen Menschen. Neben der Beratung bietet LesMigraS ebenfalls die Begleitung zu Behörden oder schwierigen Gesprächen an oder vermittelt an andere Beratungs- oder Unterstützungseinrichtungen. Auch zu Gewalt in LSBTIQ-Beziehungen, die zumeist unsichtbar ist, bietet LesMigraS Einzel- und Paarberatungen an. Des Weiteren ist die Beratungsstelle auf Gewalt und Diskriminierung durch die Polizei spezialisiert und dokumentiert Fälle von Polizeige-

LesMigraS: Stimmen arabischer Lesben - Arabische Lesben in Deutschland und Europa, Berlin 2004.

96 LesMigraS: Chronologie der Antidiskriminierungs- und Antigewaltarbeit der Lesbenberatung.

97 LesMigraS: Team, https://lesmigras.de/team.html vom 20.08.2020.

98 LesMigraS: Förderung, https://lesmigras.de/foerderung.html vom 15.09.2020.

99 Aktuell [2019] werden Beratungen in den Sprachen Englisch, Persisch, Türkisch, Niederländisch, Deutsch und deutsche Gebärdensprache angeboten. Bei Bedarf können Dolmetscher*innen für andere Sprachen hinzugezogen werden. 
walt an LBT ${ }^{*} \mathrm{I}^{*}$-Personen. ${ }^{100} \mathrm{Zu}$ rechtlichen Fragen bietet eine Rechtsanwältin kostenlose Beratung zu Themen wie Asylrecht, ${ }^{101}$ Aufenthaltsrecht oder binationale Partnerschaften an. Seit 2014 sind zudem Beratungen für Inter" -Personen oder deren Angehörige, die Gewalt oder Diskriminierung erfahren haben, möglich. ${ }^{102}$ Vor allem der Fokus auf Mehrfachdiskriminierung ist ein Alleinstellungsmerkmal der LBT* ${ }^{* *}$-Beratung von LesMigraS. ${ }^{103}$ Die Geschäftsführer* in berichtet in einem Interview, dass LesMigraS Beratungsanfragen aus ganz Deutschland bekomme. ${ }^{104}$ Die Erweiterungen der Beratungsangebote (z.B. Asyl, Polizeigewalt) stellen zumeist eine Antwort auf die Anfragen, mit denen LesMigraS in den Beratungen konfrontiert wird, dar. Das Ziel der Beratung, einen selbstermächtigten Umgang mit Gewalt und Diskriminierungserfahrungen zu erarbeiten, ist auch im Empowerment-Projekt »Tapesh« zentral.

\section{Das Empowerment-Projekt "Tapesh"}

Empowerment ist ein zentraler Fokus der Arbeit von LesMigraS. Das heißt, LesMigraS versucht mit seinen Angeboten, Menschen zur Selbstermächtigung zu unterstützen. Entsprechend wurde 2010 das Projekt »Tapesh «, das Empowerment-Projekt von LesMigraS, ins Leben gerufen. Das Projekt »Tapesh« wird unterstützt und gefördert von der »Initiative Berlin tritt ein für Selbstbestimmung und Akzeptanz sexueller Vielfalt", der Landesstelle für Gleichbehandlung gegen Diskriminierung - Fachbereich LSBTI sowie der Berliner Senatsverwaltung für Justiz, Verbraucherschutz und Antidiskriminierung. Ziel von "Tapesh« ist es, Lesben, Schwule, Bisexuelle, Trans* - und Intergeschlechtliche Menschen zu unterstützen und Handlungsstrategien für diese Personengruppen $\mathrm{zu}$ entwickeln. Dazu gehört die generelle Sensibilisierung für Gewalt und Diskriminierung in der Gesellschaft sowie die gemeinsame Entwicklung von Perspektiven und Strategien auf Basis der Stärken und Ressourcen der jeweiligen Gruppe oder der einzelnen Teilnehmer*innen. Die erarbeiteten Strategien sollen - wenn möglich - auch umgesetzt und reflektiert werden. ${ }^{105}$ Ein wichtiger Baustein von »Tapesh « sind die regelmäßigen Empowerment-Workshops und Veranstaltungen: So haben in den letzten Jahren beispielsweise Workshops zur Selbstverteidigung oder Workshops, in denen sich bestimmte Gruppen zu Themen wie Körper, Geschlecht, offene Beziehungen, Warten auf den Asylbescheid oder Trennungen austauschen, stattgefunden. Viele Workshops arbeiten auch mit kreativen Ansätzen, wie dem unsichtbaren Theater, Kunst oder kreativem Schreiben. Die Workshops werden oft in mehreren Sprachen angeboten und sind

100 Die Beratung zu Polizeigewalt sowie die Dokumentation von Polizeigewalt werden seit 2016 angeboten.

101 Seit 2016 gibt es das Projekt »Queer refugees support«, in dessen Rahmen das Beratungsangebot, aber auch das Workshop- und Veranstaltungsangebot erweitert wurde.

102 LesMigraS: Beratung, https://lesmigras.de/beratung.html vom 15.09.2020.

103 In Köln gibt es noch »baraka«, in Berlin »Cladt« und in Frankfurt a.M. eine Deutsch-iranische Beratungsstelle.

104 Saideh Saadat-Lendle: »Kategorien? Nein Danke! Saideh Saadat-Lendle über die Arbeit von LesMigraS«, in: »Nicht jammern, sondern klagen!«. Diskriminierungsschutz und Recht für Lesben, Schwule und Trans", S. 19-24. LesMigraS: Tapesh, https://lesmigras.de/tapesh.html vom 20.08.2020. 
zum Teil offen für alle LGBTIQA-Menschen, haben aber zum Teil auch spezielle Zielgruppen, wie zum Beispiel Menschen mit Flucht- und Migrationserfahrungen. ${ }^{106} \mathrm{Ne}-$ ben den Workshops bietet »Tapesh« auch andere Veranstaltungen, wie gemeinsame Brunchs oder Lesungen an. 2010, also gleich zu Beginn des Empowerment-Projektes, wurde eine große Diskussionsveranstaltung - „Verbindungen sprechen « - organisiert, bei der es speziell um Rassismus und Transphobie in der LSBTI-Szene ging. Die Beiträge aus der Veranstaltungsreihe und die Erkenntnisse aus den Workshops wurden anschließend in einer Broschüre veröffentlicht. Bildungs- und Öffentlichkeitsarbeit in Form von eigenen Broschüren und Online-Materialien ist ein weiterer Interventionsansatz von »Tapesh«. Hier wird für Gewalt und Diskriminierung sensibilisiert, es werden Handlungsstrategien vorgestellt oder Bündnis- und Vernetzungsfragen diskutiert. Ein weiterer Aspekt des Projektes ist die Präsenz von LesMigraS auf verschiedenen politischen, sportlichen wie kulturellen Veranstaltungen. Ziel ist es, an diesen Orten den Austausch mit Menschen, die von Gewalt und Diskriminierung betroffen sind, zu fördern. ${ }^{107}$ Neben den Broschüren und Online-Materialien, die im Rahmen von »Tapesh « entwickelt wurden, stellen auch andere Veröffentlichungen einen wichtigen Bestandteil der Arbeit von LesMigraS dar.

\section{Antirassistische und queer-feministische Öffentlichkeits- und Bildungsarbeit ${ }^{108}$}

Die Arbeit von LesMigraS setzt nicht nur darauf, von Gewalt Betroffene zu beraten, zu unterstützen und $\mathrm{zu}$ empowern, sondern will auch mit Öffentlichkeitsarbeit sowohl die eigene Zielgruppe als auch die Community und die Gesellschaft erreichen. Die Öffentlichkeitsarbeit kann insofern als Bildungsarbeit verstanden werden, als zu Themen wie Gewalt und Mehrfachdiskriminierung informiert und sensibilisiert wird. Darüber hinaus geht es auch darum, Gewalt- und Mehrfachdiskriminierungs-Strukturen in der Gesellschaft und der Community zu benennen und alternative Möglichkeiten des Zusammenlebens aufzuzeigen. Dieser Herangehensweise liegt ein mehrdimensionales Gewaltverständnis zugrunde, demzufolge Gewalt nicht nur in zwischenmenschlichen Beziehungen $\mathrm{zu}$ finden ist, sondern sich auch strukturell und institutionell manifestiert. ${ }^{109}$ In diesem Zusammenhang spricht LesMigraS auch davon, einen ganzheitlichen Ansatz in der Antigewalt- und Antidiskriminierungsarbeit zu verfolgen: Es reiche nicht aus, in der Antigewaltarbeit nur bei den Betroffenen anzusetzen und damit eine »Individualisierung in der sogenannten Opferhilfe« zu affirmieren. ${ }^{110}$ Ähnlich wie im Fall der Erweiterung der Beratungsthemen geht auch die Community-basierte Aufklärungsund Vernetzungsarbeit auf die Erfahrungen der jahrelangen Beratungsarbeit und die

106 LesMigraS: Workshops und Events, https://lesmigras.de/workshops_und_events.htmlvom 20.08. 2020.

107 LesMigraS: Tapesh_Angebote, https://lesmigras.de/tapesh_angebote.html vom 20.08.2020.

108 Der Fokus der folgenden Darstellung liegt vor allem auf der Öffentlichkeitsarbeit, die nicht in den Materialkorpus aufgenommen wurde.

109 LesMigraS: Gewaltrad, https://lesmigras.de/gewaltrad.html vom 20.08.2020.

110 Saideh Saadat-Lendle: Pressekonferenz zur Vorstellung erster Ergebnisse der bisher umfangreichsten Studie zu Gewalt- und Mehrfachdiskriminierungserfahrungen von lesbischen/bisexuellen Frauen und Trans*. LesMigraS 2012, https ://lesmigras.de/tl_files/lesmigras/pressemitteilung en/Redebeitrag\%20Saideh\%20Saadat-Lendle.pdf vom 20.08.2020. 
Ergebnisse eigener Studien zurück. Sie ist eine Antwort darauf, dass Menschen, die Gewalt und Mehrfachdiskriminierung erfahren, sich primär Unterstützung im eigenen sozialen Umfeld suchen. Entsprechend wird die Community als ein Ort verstanden, der potentiell unterstützend ist, der allerdings Gewalt- und Diskriminierungserfahrungen auch verstärken kann. ${ }^{111}$ Sowohl in Bezug auf die Community und Gesellschaft als auch auf individueller Ebene bleibt die Öffentlichkeitsarbeit meist nicht dabei stehen, Gewaltstrukturen $\mathrm{zu}$ benennen und offenzulegen, sondern bietet immer auch mögliche Handlungsstrategien an. Bildungsarbeit wird so nicht nur als Aufgabe verstanden, über Missstände aufzuklären, sondern auch als Ort, an dem Möglichkeiten offengelegt werden, auf Gewalt- und Mehrfachunterdrückung zu reagieren.

Im Zuge dieser Öffentlichkeits- und Bildungsarbeit hat LesMigraS in den letzten 25 Jahren zahlreiche Broschüren, Studien, Bücher, Pressemitteilungen, Redebeiträge, Filme, Flyer, Plakate und Online-Texte herausgegeben. Viele der umfangreicheren Veröffentlichungen (Studien, Broschüren und Bücher) werden projektbezogen finanziell gefördert. Die Veröffentlichungen liegen vor Ort bei LesMigraS aus, können innerhalb von Berlin kostenlos bestellt werden und sind zumeist auch online zugänglich. Broschüren erscheinen seit 2004 regelmäßig. In vielen dieser Broschüren geht es darum, die Lebensrealitäten von Menschen an den Schnittstellen von Migration, Flucht und queeren Lebensweisen sichtbar zu machen. $\mathrm{Zu}$ den ersten veröffentlichten Broschüren gehören vier im Rahmen des Aktionsprogramms der Europäischen Union zur Bekämpfung von Diskriminierung veröffentlichte Bücher, in denen über die Lebensrealitäten von arabischen, russischen, türkischen und iranischen Lesben in Europa berichtet wird. ${ }^{112}$ Im Jahr 2017 schließt die Broschüre "10 PORTRÄTS« wieder an dieses Format an. In mehreren Sprachen werden auch hier die Geschichten von lesbischen, schwulen, bisexuellen und Trans*-Geflüchteten in Deutschland erzählt. ${ }^{113}$ Neben solchen Broschüren, die versuchen, unsichtbare Lebensrealitäten sichtbarer zu machen, veröffentlicht LesMigraS auch Broschüren, die für Gewalt und Diskriminierungen sensibilisieren und gleichzeitig Handlungsmöglichkeiten aufzeigen. In diesem Zusammenhang wären beispielsweise die beiden Broschüren »Verbindungen sprechen ${ }^{114}$ und »Unterstützung geben ${ }^{115} \mathrm{zu}$ nennen, die $2011 \mathrm{im}$ Rahmen von »Tapesh« erschienen sind, aber auch das

111 LesMigraS: Gewaltverständnis, https://lesmigras.de/tl_files/lesmigras/Tapesh/Texte/2015_Cewaltv erstaendnis.pdf vom 26.08.2020.

112 LesMigraS: Russische Lesben in Europa, Berlin 2004; LesMigraS: Stimmen arabischer Lesben - Arabische Lesben in Deutschland und Europa; LesMigraS: Leben iranischer Lesben - Iranische Lesben in Deutschland und Europa, Berlin 2006; LesMigraS: Zeichen setzen! Türkeistämmige Lesben in Deutschland und Europa, Berlin 2004.

113 LesMigraS: 10 PORTRÄTS - Lesbische, Schwule, Bisexuelle und Trans* Geflüchtete in Deutschland. 2017.

114 LesMigraS: Verbindungen sprechen. Empowerment in Bezug auf Rassismus und Transphobie in LSBTI-Kontexten 2011, https://lesmigras.de/tl_files/lesmigras/Tapesh/LM_Broschuere_Tapesh_einz elseiten_korrigiert.pdf vom 20.08.2020.

115 LesMigraS: Unterstützung geben. Handlungsstrategien im Umgang mit Gewalt und Diskriminierung. 2011, https://lesmigras.de/tl_files/lesmigras/Tapesh/LM_Broschuere_Tapesh_Unterstuetzung Geben.pdf vom 20.08.2020. 
2018 erschienene Arbeitsbuch »GRENZEN SETZEN! «. ${ }^{116}$ Die Broschüren, die zu den umfangreicheren Veröffentlichungen von LesMigraS zählen, leisten insofern Bildungsund Aufklärungsarbeit, als sie für die verschiedenen Lebensrealitäten mehrfachdiskriminierter Menschen sensibilisieren und so diese sichtbar machen. Gleichzeitig geht es auch darum, konkrete Gewalt und Mehrfachdiskriminierung offenzulegen, zu benennen und, sowohl auf struktureller und institutioneller als auch auf zwischenmenschlicher und individueller Ebene, Handlungsstrategien aufzuzeigen. Ein weiteres wichtiges Instrument der Öffentlichkeits- und Bildungsarbeit stellen Pressemitteilungen, Stellungnahmen und Redebeiträge dar. Bei diesen ist die Zielgruppe vor allem die Community und die Gesellschaft. Auch hier geht es darum, für Rassismus und Transphobie zu sensibilisieren, gewaltsame Strukturen zu benennen und alternative Möglichkeiten aufzuzeigen.

Ein weiterer zentraler Aspekt der Bildungs- und Aufklärungsarbeit von LesMigraS ist die Durchführung und Veröffentlichung von eigenen Studien. In diesen Studien geht es zumeist darum, fundiertes Wissen in Bezug auf Gewalterfahrungen und Mehrfachdiskriminierungen zu generieren und öffentlich zu machen. Damit gelingt es zum einen, Lebensrealitäten zu erfassen und sichtbar zu machen, denen sonst zumeist kein Raum in wissenschaftlichen Studien zukommt, zum anderen stellen die Ergebnisse dieser Studien eine wichtige Reflexionsgrundlage für die Arbeit von LesMigraS dar. Zudem dienen die erhobenen Forschungsdaten auch als Argumentationsquelle für gesellschaftspolitische Forderungen. Bei den bisher durchgeführten Studien handelt es sich zumeist um geförderte Projekte, die mitunter auch in Kooperation mit anderen Einrichtungen oder Wissenschaftler*innen stattfinden. Oft schließen sich Kampagnen, Broschüren und Veranstaltungen an die durchgeführten Studien an. Eines der ersten Projekte von LesMigraS als eigener Arbeitsbereich war die Mitarbeit an dem von 2000 bis 2002 stattfindenden EU-Projekt »Gewalt gegen Lesben«, in dem zunächst eine quantitative Studie zu psychosozialen Beratungsangeboten für Lesben in Deutschland, Österreich und Belgien durchgeführt wurde. Die Studie wurde von einer Plakatkampagne - »Ich habe nichts gegen Lesben, ABER ...« - begleitet. ${ }^{117}$ Die Plakate wurden in Wien, Frankfurt, Köln, Neubrandenburg und Kassel ausgehängt, um auf alltägliche Diskriminierungen gegen Lesben aufmerksam zu machen. ${ }^{118}$ Neben diesen ersten Erhebungen in der Anfangszeit wurde 2010 bis 2012 die mit Abstand größte wissenschaftliche Studie von LesMigraS durchgeführt. Die Studie »... nicht so greifbar und doch real« fand im Rahmen der Kampagne zu Gewalt- und Mehrfachdiskriminierungserfahrungen von lesbischen, bisexuellen Frauen und Trans*-Menschen in Deutschland statt, deren drei Säulen Öffentlichkeitsarbeit, Vernetzung und Beratungsarbeit waren. Die Studie umfasste einen

116 LesMigraS: Grenzen setzen - Ein Arbeitsbuch 2017, https://lesmigras.de/tl_files/lesmigras/Texte/L esMigraS_Grenzen_Setzen_online.pdf.

117 LesMigraS: Chronologie der Antidiskriminierungs- und Antigewaltarbeit der Lesbenberatung.

118 Constance Ohms/Karin Müller: Gut aufgehoben? Zur psychosozialen Versorgung lesbischer Frauen mit Gewalt und/oder Diskriminierungserfahrungen im europäischen Vergleich 2001, https:/ /lesmigras.de/tl_files/lesbenberatung-berlin/Gewalt\%20(Dokus,Aufsaetze...)/gut_aufgehoben.pdf vom 20.08.2020. 
Fragebogen, der von 2143 Menschen ausgefüllt wurde, sechs Interviews und eine Gruppendiskussion. Der Fokus der Studie lag auf Aspekten der Mehrfachdiskriminierung aufgrund von Rassismus, Sexismus und Transphobie. Sie wurde deutschlandweit von Antigewalt- und Antidiskriminierungsstellen sowie LSBTIQ-Projekten unterstützt. ${ }^{119}$ Die Ergebnisse wurden 2012 auf einer Fachtagung und einer Pressekonferenz vorgestellt und diskutiert. Die Studie ist vollumfänglich online verfügbar. Zudem gibt es von LesMigraS zusammenfassende Aufarbeitungen der Ergebnisse, sowie Ergebnisse und Empfehlungen zu den Bereichen Politik, Bildung, Arbeit, öffentlicher Raum, Familie und Verwandte, Freizeit und Dienstleistungen, öffentliche Ämter und Behörden, Beratungs- und Antidiskriminierungs-Antigewaltstellen. ${ }^{120}$ Wissenschaftliche Studien/Umfragen stellen so ein wichtiges Mittel dar, um den Bedarf im eigenen Arbeitsbereich zu erheben, um Empfehlungen und Forderungen an die Gesellschaft und die LSBTIQ*-Community weiterzugeben, aber auch um Mehrfachdiskriminierungen sichtbarer zu machen.

\subsection{Jugendnetzwerk Lambda Berlin-Brandenburg}

Das Jugendnetzwerk Lambda Berlin Brandenburg kann als ein sehr vielseitiges Projekt beschrieben werden, das seit 2006 von Jahr zu Jahr wächst und sich mittlerweile als queeres Jugendzentrum institutionalisiert hat. Dabei sind die vielen neuen Projekte und die Institutionalisierung auch Errungenschaften der Kämpfe, die das Jugendnetzwerk als Interessenvertretung der LSBTIQ-Jugendlichen in Politik und Öffentlichkeit führt. Immer wieder wird in Form von Pressemitteilungen öffentlichkeitswirksam Stellung bezogen. Als Jugendverband und mit hauptamtlichen Mitarbeiter*innen bietet das Jugendnetzwerk den Jugendlichen Strukturen, um sich gegenseitig zu unterstützen. Seit dem Einzug ins queere Jugendhaus, das groß und barrierefrei ist, kann Lambda $B B$ zudem vermehrt Orte des Beisammenseins und Austauschs schaffen. Auf Basis des peer-to-peer-Ansatzes leisten viele ehrenamtliche Jugendliche wichtige Aufklärungs-, Beratungs- und Leitungsarbeiten für andere Jugendliche. Dabei werden die beratenden und aufklärenden Jugendlichen selbst wiederum von hauptamtlichen Mitarbeiter*innen unterstützt und begleitet. Die Jugendlichen sind aber auch mit dabei, wenn ihre Interessen beispielsweise am »Runden Tisch« oder bei einem Treffen mit dem Senat oder sonstigen Vertreter*innen der Politik und Gesellschaft vertreten werden.

119 LesMigraS: >... nicht so greifbar und doch reak. Eine quantitative und qualitative Studie zu Cewalt- und (Mehrfach-)Diskriminierungserfahrungen von lesbischen, bisexuellen Frauen und Trans* in Deutschland, Berlin 2012, https://lesmigras.de/tl_files/lesbenberatung-berlin/Gewalt\%2 o(Dokus,Aufsaetze...)/Dokumentation\%20Studie\%20web_sicher.pdf vom 20.08.2020.

120 LesMigraS: Studie, LesMigraS, https://lesmigras.de/studie_mehrfachdiskriminierung.html vom 27.08.2020. Eine etwas kleinere Studie hat 2015 stattgefunden. Hier hat LesMigraS in verschiedenen Aufnahmeeinrichtungen und Beratungsstellen in Berlin eine Umfrage zur Situation von Asylsuchenden LSBTIQ gemacht und im Anschluss eine Stellungnahme zu diesem Thema veröffentlicht. LesMigraS: Stellungnahme zur Situation von asylsuchenden LSBTIQ in Erstaufnahmeeinrichtungen und Gemeinschaftsunterkünften in Berlin 2015. 


\section{Die drohende Insolvenz}

Das Jugendnetzwerk Lambda wurde im März 1990, also kurz vor der Wiedervereinigung, in der DDR als Jugendverband gegründet. Ziel war es, die Interessen von schwulen, lesbischen und bisexuellen Jugendlichen am »Runden Tisch « der Jugend zu vertreten. ${ }^{121}$ Nach der Wiedervereinigung war Lambda Berlin Brandenburg zuständig für das ganze Bundesgebiet und damit sowohl in der ehemaligen DDR als auch in Westdeutschland die erste Interessenvertretung für LSB-Jugendliche. Mittlerweile ist das Jugendnetzwerk Lambda BB einer von sechs Bundesverbänden, die unter dem Dach der Bundesgeschäftsstelle in Erfurt vereint sind. Die Bezeichnung Lambda bezieht sich auf den griechischen Buchstaben $\lambda$, der vor allem in der $\mathrm{DDR}^{122}$ ein verbreitetes Zeichen für schwules und lesbisches Leben war. Das Symbol hat seinen Ursprung im englischsprachigen Raum, in dem das L für »Liberty«, also Freiheit, seht.

Seit der Gründung unterlag das Jugendnetzwerk Lambda Berlin-Brandenburg ständigen Veränderungsprozessen: Die Einrichtung musste mehrfach umziehen, es wurden Cafés eröffnet und wieder geschlossen und die verschiedensten Beratungs- und Aufklärungsprojekte realisiert, eingestellt und wiederaufgebaut. So sind in den ersten 14 Jahren eine eigene Bibliothek, ein Aufklärungsprojekt, ein Beratungsprojekt, betreutes Wohnen, das »Lambda ${ }^{2}$ «-Projekt für schwule, lesbische und bisexuelle Jugendliche mit Behinderung, ein kommerzielles Café und verschiedene Jugendgruppen entstanden. Nachdem immer mehr Jugendliche aus Brandenburg die Angebote in Anspruch nahmen, wurde im Jahr 2001 das ursprüngliche Lambda Berlin schließlich erweitert zu Lambda-Berlin-Brandenburg. Aufgrund von Misswirtschaft der damaligen Geschäftsführung stand das Jugendnetzwerk allerdings ab 2004 kurz vor der Insolvenz. Die eigenen Räumlichkeiten mussten aufgegeben und viele Projekte gekürzt beziehungsweise an andere Träger*innen abgegeben werden. ${ }^{123}$ In den nächsten zwei Jahren (2004-2006) beschränkte sich die Arbeit von Lambda BB auf kleinere Projekte und darauf, die Insolvenz abzuwenden. ${ }^{124}$ Mit neuer Geschäftsführung und zehn verbliebenen ehrenamtlichen Mitarbeitern konnte das Jugendnetzwerk Lambda BB ab 2006 erneut starten. Mit der Zeit rief Lambda BB viele der - in der Krise verlorenen - Projekte wieder ins Leben, und zahlreiche neue Projekte kamen hinzu. Inzwischen ist das Projekt zu einem queeren Jugendzentrum angewachsen. Auch die finanziellen Förderungen und personellen Ressourcen haben sich in den letzten Jahren verändert. Die Einrichtung verfügte zumeist über eine hauptamtliche Geschäftsführung, später kam eine Teilzeitstelle für eine pädagogische/psychologische Fachkraft hinzu. Durch die Berliner Senatsverwaltung wurde das Projekt in den letzten dreißig Jahren teilfinanziert. ${ }^{125}$ Zusätzlich verfügte Lambda

Bis heute wird diese Tradition aufrechterhalten und Vertreterinnen aus der Politik und anderen Orten an den Runden Tisch geladen, wenn es um die Interessenvertretung der Jugendlichen geht.

Die in Westdeutschland verwendete Regenbogenfahne war in der DDR nicht so weit verbreitet wie das $\lambda$-Symbol.

In dieser Zeit ist beispielsweise »ABqueer«entstanden, das das Beratungsprojekt»In und Out«und das Aufklärungsprojekt von Lambda BB übernommen haben.

124 Das Jugendnetzwerk hatte 30.000 Euro Schulden.

125 Jugendnetzwerk Lambda:BB: Unsere Geschichte, https://www.lambda-bb.de/uber-uns/unsere-ges chichte vom 20.08.2020. 
$B B$ durch Fundraising und Drittmittelfinanzierung über Eigenmittel. ${ }^{126}$ Im Jahr 2011 gelang es Lambda BB nach Verhandlungen mit dem Senat, zusätzliche finanzielle Mittel aus den Töpfen der »Initiative Berlin tritt ein für Selbstbestimmung und Akzeptanz sexueller Vielfalt« zu erwerben, die für das Demokratieprojekt »queer@school« zur Verfügung gestellt wurden. ${ }^{127}$ Seit 2018 ist Lambda BB offiziell ein queeres Jugendzentrum und mit entsprechender Finanzierung ausgestattet. ${ }^{128}$ Neben den Räumen, die den Jugendlichen als Treffpunkt und zum Austausch zur Verfügung stehen, sind die Beratungen und einzelne Projekte zentraler Bestandteil von Lambda BB. Lambda BB versteht sich aber nach wie vor auch als politische Interessenvertretung von Jugendlichen. Inhaltlich basiert die Arbeit des Jugendnetzwerks Lambda Berlin-Brandenburg auf vier Säulen:
a) Beratung,
b) Bildungs-, Öffentlichkeits- und Lobbyarbeit,
c) Empowerment,
d) Kultur und Freizeit.

\section{Ein queeres Jugendhaus}

Das Jugendnetzwerk Lambda BB ist ein Raum, an dem die Jugendlichen nicht nur beraten werden, sondern in dem sie sich auch treffen, austauschen oder gemeinsam ihre Freizeit verbringen können. So hat es in den Jahren seit der Gründung dort immer wieder ein Café gegeben, als Ort des informellen Austausches, der aber auch für regelmäßige Treffen von verschiedenen Gruppen genutzt wurde. ${ }^{129}$ Zurzeit [Stand: 2018] treffen sich sechs verschiedene Gruppen unter dem Dach des queeren Jugendzentrums von Lambda BB. Die Gruppen bieten Austausch, Diskussionen und gemeinsame Freizeitaktivitäten an und unterscheiden sich hauptsächlich in Bezug auf die Zielgruppe und entsprechend auch auf die Themen, die zentral gesetzt werden. Die jugendlichen Gruppenleiter*innen treffen sich regelmäßig, um sich über Probleme und Schwierigkeiten auszutauschen, aber auch für Fortbildungen. ${ }^{130}$ Weil die Beratungs- und Austauschräume häufig genutzt werden, hatte Lambda BB schon immer mit einem Mangel an räumlichen Ressourcen zu kämpfen. Entsprechend war es lange Zeit eine zentrale Aufgabe des Jugendnetzwerks, im Interesse des Projekts und der Jugendlichen größere Räume, aber auch eine strukturell abgesicherte queere Jugendarbeit in Form eines Jugendzentrums in Berlin einzufordern. Die Forderungen nach größeren Räumlichkeiten wurden 2014 erfüllt. Lambda BB konnte in die größeren und barrierefreien Räumlichkeiten des ehemaligen Jugendzentrums »Freitem ${ }^{131}$ im Bezirk Pankow einziehen. Die

126 Diese Angaben gelten für die Jahre 2006 bis 2012.

127 Jugendnetzwerk Lambda:BB: Unsere Geschichte.

128 Jugendnetzwerk Lambda:BB: Zur Vergabe des queeren Jugendzentrums an Lambda BB 2020, https://www.lambda-bb.de/wp-content/uploads/2018/06/2018-06-08-Pressemitteilung-Verg abe-queeres-Jugendzentrum.pdf vom 20.08.2020.

129 Jugendnetzwerk Lambda:BB: Unsere Geschichte.

130 Jugendnetzwerk Lambda:BB: Jugendgruppen, https://www.lambda-bb.de/jugendgruppen vom 20.08.2020.

131 Aufgrund von Einsparungen der Stadt Berlin ist es zur Schließung des Jugendzentrums gekommen. 
Räumlichkeiten wurden und werden dem Jugendnetzwerk Lambda BB zum Betriebskostenpreis zur Verfügung gestellt. ${ }^{132}$ Mit dem Umzug steht der Einrichtung genügend Platz für Beratung und Gruppenangebote zur Verfügung. Darüber hinaus konnte in den neuen Räumen mit dem »Pride Café«, das ehrenamtlich von den Jugendlichen betrieben wird, erneut ein niederschwelliger Treffpunkt und Austauschort für Jugendliche geschaffen werden. ${ }^{133}$ Mit der Nutzung der Räumlichkeit geht die Verpflichtung einher, zweimal sechs Stunden pro Woche offene Jugendarbeit anzubieten. Entsprechend führte die Eröffnung des Jugendhauses auch zu einer Öffnung der Zielgruppe. Es werden aber nach wie vor Schutzräume für LSBTIQ-Jugendliche bereitgestellt. Abgesehen von den Räumlichkeiten und den Aufgaben der offenen Jugendarbeit betonte die Einrichtung nach dem Einzug ins Jugendzentrum allerdings, dass sie nach wie vor ein Jugendverband und kein - mit entsprechenden Mitteln ausgestattetes - Jugendzentrum sei. Es fehlten personelle und finanzielle Ressourcen, um den Bedarf an einem queeren Jugendzentrum decken zu können. ${ }^{134}$ Schließlich wurden auch die Forderungen nach einem queeren Jugendzentrum, das mit »bedarfsgerechten Mitteln« ausgestattet ist, im September 2018 erfüllt. Bereits 2016 war die Eröffnung eines queeren Jugendzentrums im Koalitionsvertrag der rot-rot-grünen Stadtregierung festgeschrieben worden. ${ }^{135}$ Nachdem sich Lambda BB, neben anderen Gruppen und Träger*innen, an der Erstellung des Konzepts für das Jugendzentrum beteiligt hatte, ging im Juni 2018 die Vergabe der Förderung für das queere Jugendzentrum an das Projekt. ${ }^{136}$ Im September 2018, über vier Jahren nach dem Einzug in das queere Jugendhaus, konnte offiziell und unter der Leitung von Lambda BB ein queeres Jugendzentrum eröffnet werden, das nun im Aufbau ist und weitere Beratungsgruppen, aber auch Freizeitangebote für eine breite Zielgruppe möglich macht. ${ }^{137}$ Neben Café, Bibliothek und Gruppentreffen finden in den Räumen von Lambda BB auch Beratungen statt.

\section{Die peer-to-peer-Beratung}

Die Beratung des Jugendnetzwerks Lambda BB umfasst die klassische »In und Out Beratung«, die spezifische Trans*-Beratung sowie das Beratungsprojekt »Lambda ${ }^{2}$. Bereits seit 1995 bietet Lambda Beratungen von Jugendlichen für Jugendliche (peer to peer) an. Das »Lambda ${ }^{2}$ «-Beratungsprojekt mit speziellem Fokus auf schwule, lesbische und Trans*-Jugendliche mit Handicap wurde vier Jahre später ins Leben gerufen. Auch Trans*-Menschen als spezielle Zielgruppe wurden 2001 in Form der

132 Jugendnetzwerk Lambda:BB: Queeres Jugendhaus in Pankow! 2014, https://www.lambda-bb.de/w p-content/uploads/2013/09/Pressemitteilung-04-2013.pdf vom 27.08.2020.

133 Jugendnetzwerk Lambda:BB: Pride Café, https://www.lambda-bb.de/pridecafe vom 20.09.2020.

134 Jugendnetzwerk Lambda:BB: ... Eröffnung erstes queeres Jugendhaus in Berlin! 2014, https://www .lambda-bb.de/wp-content/uploads/2014/04/Pressemitteilung-02-2014.pdf vom 20.08.2020.

135 Jugendnetzwerk Lambda:BB: Berliner Senat beschließt Konzept für queeres Jugendzentrum! 2018, https://www.lambda-bb.de/wp-content/uploads/2018/02/2018-02-16-PM-Konzept-QJZ .pdf vom 15.09.2020.

136 Jugendnetzwerk Lambda:BB: Zur Vergabe des queeren Jugendzentrums an Lambda BB.

137 Jugendnetzwerk Lambda:BB: Queeres Jugendzentrum, https://www.lambda-bb.de/projekte/quee res-jugendzentrum vom 20.08.2020. 
»s/he Transberatung « berücksichtigt. ${ }^{138}$ In Jahren der drohenden Insolvenz wurde das Beratungsprojekt "In und Out« für kurze Zeit an den neu gegründeten Verein »ABqueer e.V.« 2005 abgegeben. Nachdem Lambda BB die finanzielle Krise einigermaßen überstanden hatte, konnte Mitte 2006 das Beratungsprojekt "In und Out « wieder übernommen werden. Gleiches gilt für das integrative Beratungsprojekt »Lambda ${ }^{2}$; vier Jahre später wurde es wieder Teil des Lambda-Angebotes. ${ }^{139}$ »In und Out « ist mittlerweile ein bundesweites peer-to-peer-Beratungsprojekt, das durch die Mittel des Bundesministeriums finanziert wird und in den Räumen von Lambda BB angesiedelt ist. Das heißt, die Räume des Jugendnetzwerks Lambda BB werden für Treffen und Beratung genutzt, die Beratung findet aber bundesweit statt. Das jugendliche Beratungsteam wird von bei Lambda BB angestellten Psycholog*innen begleitet und auch ausgebildet, es gibt Supervisionen, Teamsitzungen und Fortbildungen. Bei Bedarf unterstützen die Psycholog*innen aber auch die Ratsuchenden. Zielgruppe der Beratungen sind Jugendliche bis 27, die lesbisch, bisexuell, schwul, trans* oder queer sind. Es können sich aber auch Bezugspersonen an die »In und Out Beratung « wenden. Neben der Möglichkeit persönlicher Beratungsgespräche und einer »In und Out«-Sprechstunde gibt es E-Mail- und Chat-Beratungen. ${ }^{140}$ Nach Absprache bietet das junge Team auch Telefonberatung an. Wie der Name der Beratung, aber auch die Homepage der »In und Out Beratung « nahelegt, stehen Fragen zum »inneren« und »äußeren« Coming-out im Mittelpunkt der Beratung. Es können aber auch Fragen zu Sexualität, Beziehungen, Problemen mit den Eltern, Mehrfachdiskriminierungen und anderen Themen besprochen werden. ${ }^{141}$ Im Rahmen des "Lambda ${ }^{2}$ «-Projekts wird eine spezielle Beratungsmöglichkeit für lesbische, schwule, Trans* - und bisexuelle Jugendliche mit Handicap angeboten. Auch hier kann auf E-Mail-, Telefon- oder persönliche Beratung zurückgegriffen werden. Diese Beratung findet allerdings nicht im Sinne des peer-to-peer-Ansatzes statt, sondern wird von einer pädagogischen/psychologischen Fachkraft durchgeführt. ${ }^{142}$ Das Jugendnetzwerk Lambda BB ist aber nicht nur innerhalb der eigenen Räume aktiv, sondern versucht auch jenseits davon, queere Jugendliche zu erreichen. Im Rahmen der »LesBiSchwulen Bus T*our « wird unter anderem die »In und Out Beratung« nach Brandenburg gebracht.

\section{Die "LesBiSchwule Bus T*our «: Den ländlichen Raum erobern}

»LesBiSchwule Bus $T^{*}$ our « gibt es bereits seit 1998. Sie fand zunächst unter der Trägerschaft des Vereins »andersartig e.V.« statt. Seit 2006 ist das Jugendnetzwerk Lambda BB Partner* des Projektes und unterstützt es durch die Teilnahme der Ehrenamtler*innen, aber auch finanziell. Ihren Anfang nimmt die »LesBiSchwule Bus T*our« in der Idee,

138 Die Trans ${ }^{*}$-Beratung ist dann einfach in die $»$ In und Out «- und »Lambda ${ }^{2} «-B e r a t u n g ~ e i n g e g l i e d e r t$ worden.

139 Jugendnetzwerk Lambda:BB: Unsere Geschichte.

140 Einzel-und Gruppenchats.

141 Jugendnetzwerk Lambda:BB: In\&Out-Beratung, https://comingout.de/ vom 20.08.2020.

142 Jugendnetzwerk Lambda:BB: Lambda² Projekt, https://www.lambda-bb.de/projekte/lambda\%c2 \%b2-projekt vom 20.08.2020. 
den CSD auch nach Brandenburg zu bringen. ${ }^{143}$ Ziel der Tour ist es, die Akzeptanz von schwulen, lesbischen und Trans*-Lebensweisen im ländlichen Raum zu fördern. Einmal im Jahr reisen Aktivist*innen ${ }^{144}$ mit ihrem Bus durch Brandenburg und organisieren an verschiedenen Orten kulturelle Veranstaltungen, Workshops, Infostände sowie Aufklärungs- und Diskussionsveranstaltungen, um mit den Städten und Bürger"innen ins Gespräch zu kommen. Dabei setzen sie auf die Unterstützung schwuler, lesbischer und Trans*-Jugendlicher vor Ort. Auch das »In und Out«-Beratungsteam von Lambda $B B$ bietet eine mobile Beratung für Jugendliche, Freunde und Angehörige an. Als Zeichen der Anwesenheit wird am Rathaus die Regenbogenfahne gehisst. Gefördert wird das Projekt durch Lottomittel aus dem Ministerium für Arbeit, Soziales, Gesundheit, Frauen und Familie des Landes Brandenburg sowie durch Spendengelder. ${ }^{145}$ Mittlerweile begleiten auch einige Veröffentlichungen das Projekt. So hat 2012 die Filmemacher*in Kathrin Schulz die Tour mit der Kamera begleitet und einen Dokumentarfilm zu dem Projekt produziert. Zudem wurde extra für die Bustour die »Tour-Fibel« verfasst, in der die wichtigsten Symbole und Abkürzungen sowie die Geschichte der Community erklärt werden. ${ }^{146}$ Mit der »LesBiSchwule Bus T*our gelingt es zusätzlich zur Aufklärungs- und Beratungsarbeit im urbanen Raum, diese auch in ländlichere Gegenden zu bringen. Ein weiteres Projekt, das jenseits der Lambda BB Räume stattfindet, ist das »queer@school«-Projekt.

\section{Das Demokratieprojekt »queer@school«}

Das »queer@school«-Projekt ist finanziert durch die 2009 im Berliner Abgeordnetenhaus beschlossene Initiative "Berlin tritt ein für Selbstbestimmung und Akzeptanz sexueller Vielfalt (ISV)«. Die Förderungen sind eigenen Angaben zufolge Ergebnis der Lobby- und Öffentlichkeitsarbeit der hauptamtlichen Mitarbeiter*innen, die sich seit den Kürzungen 2006 verstärkt um finanzielle Ressourcen bemühten. Die im Zuge des Projektes stattfindenden Workshops können über den Senat abgerechnet werden, wobei die Gelder dem Bedarf der Arbeit des Jugendnetzwerks Lambda BB und dem Demokratieprojekt »queer@school«zur Verfügung stehen, die Jugendlichen aber ehrenamtlich arbeiten. Das Projekt besteht seit Frühjahr 2011 und hat das Ziel, Schüler*innen für Diskriminierung zu sensibilisieren und sie zu bestärken. Auch hier sind es im Sinne

143 Anfangs wurde der CSD in verschiedenen Städten in Brandenburg wie Cottbus, Potsdam oder Frankfurt an der Oder veranstaltet. Dieses Konzept funktionierte gut, hatte aber nicht die Reichweite, die den Aktivist*innen vorschwebte. LSBTIQ-Lebensweisen sollten an allen Orten, vor allem auch in der ländlichen Gegend in Brandenburg sichtbar werden. So entstand dann 1998 die CSDTour, die immer im Anschluss an den Berliner CSD durch Brandenburg reiste. Nachdem aufgrund der Fußball-Weltmeisterschaft 2006 die Tour später in den Sommer verschoben wurde, wurde sie in »LesBiSchwule Bus T*our « umbenannt.

144 Das Team besteht nicht nur aus Aktivist*innen von »andersartig e.V.« und Lambda BB, sondern auch aus anderen Projekten, Netzwerken und Koordinierungsstellen.

145 Landesverband AndersARTiC e.V.: Die LesBI*Schwule T*our. Die Kampagne, http://brandenburg-b leibt-bunt.de/die-kampagne.html vom 27.08.2020.

146 LesBi*SchwuleT*our: Tour-Fibel, http://brandenburg-bleibt-bunt.de/die-tour-fibel/die-regenbogen fahne.html vom 20.08.2020. 
des peer-to-peer-Ansatzes die Jugendlichen selbst, die in die Schule gehen, um aufzuklären. Die ehrenamtlichen Jugendlichen werden durch eine hauptamtliche Mitarbeiter*in begleitet. In dem Projekt geht es darum, in einem Umfeld zu intervenieren, in dem Jugendliche besonders viel Zeit verbringen. Ziel ist es, in dem für die Jugendlichen oft sehr wichtigen Raum Schule Sensibilisierungs- und Empowermentarbeit zu leisten, indem Begriffe erläutert werden, über queere Lebensweisen berichtet und für Diskriminierungen sensibilisiert wird. Zielgruppen sind »Bezirksschüler_innen-Ausschüsse, Schulklassen und allgemein Gruppen mit Menschen unter 27 Jahren «. ${ }^{147}$ Neben den Workshops mit den Schüler*innen begleiten die »queer@school«-Ehrenamtler*innen auch Treffen von Gruppen, die sich gegen Diskriminierungen einsetzen möchten. Jugendliche, die an ihrer Schule Diskriminierungen erfahren, können sich ebenfalls an das Team wenden. Das »queer@school«-Team hat zudem Flyer, Plakate, Postkarten sowie eine Wanderausstellung veröffentlicht, die auf der Homepage von Lambda BB bestellt werden können. Auf der eigenen Projekt-Homepage sind zudem noch ein queeres Lexikon und ein »Selbstverständnis« der für das Projekt relevanten Konzepte zu finden. ${ }^{148}$

\section{Öffentlichkeitsarbeit}

Das Jugendnetzwerk Lambda BB ist auch daran beteiligt, alternative Öffentlichkeiten herzustellen. So haben sich viele der Forderungen nach mehr Räumen und finanziellen Ressourcen in Pressemitteilungen niedergeschlagen. Pressemitteilungen werden aber auch genutzt, um sich zu aktuellen Community-Themen zu positionieren. ${ }^{149}$ Darüber hinaus produzieren die Jugendlichen Filme ${ }^{150}$ und Podcasts, ${ }^{151}$ die der Aufklärung und Sichtbarmachung von Lebensrealitäten dienen - dazu gibt es Bildungs- und Aufklärungsmaterialien sowie Flyer und Sticker. ${ }^{152}$ Lambda BB hat zudem drei Studien zur Diskriminierungssituation von queeren Jugendlichen - eine davon auch zu queeren Jugendlichen mit Behinderung - durchgeführt. ${ }^{153}$ Als Bundesverband veröffentlicht Lambda BB zudem eine eigene Zeitschrift - die »Out!«-, in der queere Jugendliche füreinander schreiben.

\footnotetext{
147 Jugendnetzwerk Lambda:BB: queer@school, https://queer-at-school.de/ vom 20.08.2020.

148 Ebd.

149 Jugendnetzwerk Lambda:BB: Pressemitteilungen, https://www.lambda-bb.de/presse-material/pre ssematerial vom 20.08.2020.

150 Jugendnetzwerk Lambda:BB: Filmprojekte, https://www.lambda-bb.de/presse-material/filmproje kte vom 20.08.2020.

151 Jugendnetzwerk Lambda:BB: Podcasts, https://www.lambda-bb.de/presse-material/podcasts vom 20.08.2020.

152 Jugendnetzwerk Lambda:BB: Printmaterialien, https://www.lambda-bb.de/presse-material/print materialien vom 20.08.2020.

153 Jugendnetzwerk Lambda:BB: Studien, https://www.lambda-bb.de/studien-2 vom 20.08.2020.
} 


\subsection{TransInterQueer e.V. (TrI0)}

TransInterQueer e.V. ist Interessenvertretung und Unterstützungsraum für Inter*und Trans*-Personen. Als Raum für Inter*- und Trans*-Personen bietet TrIQ verschiedenste Formen des Austauschs und Beisammenseins. Hier können Trans* - und Inter*-Menschen voneinander und miteinander lernen oder einfach jenseits von Stigmatisierungen Zeit miteinander verbringen. Die Beratung von und für Trans*- und Inter*-Menschen bietet Unterstützung jenseits von Normierung und Pathologisierung. Als Interessenvertretung bezieht TrIQ regelmäßig Stellung zu normierenden und pathologisierenden Richtlinien in der Medizin oder im Recht. TrIQ sensibilisiert mit den politischen Stellungnahmen und Broschüren aber auch auf der Ebene der Community, der Gesellschaft und bei Personen, die im Gesundheitsbereich tätig sind. ${ }^{154}$

\section{Lücken schließen und Brücken bauen}

Die Entstehung von TrIQ ist in gewisser Weise an die Geschichte des Jugendnetzwerks Lambda BB gekoppelt. Nachdem sich im Jahr 2004 - also in der Zeit, in der Lambda BB in einer finanziellen Krise steckte - aus dem Jugendnetzwerk Lambda BB heraus der Verein »ABqueer« formierte, ist wiederum zwei Jahre später aus den Räumen von »ABqueer« der Verein TransInterQueer e.V. entstanden. ${ }^{155} \mathrm{Zu}$ Beginn trafen sich etwas mehr als ein Dutzend Menschen in einem Hinterzimmer in Berlin-Neukölln, um TrIQ als »ersten Verein von und für Trans*, Inter* und Queere Menschen in Berlin, für den es damals auch in anderen europäischen Ländern noch kein Vorbild gab«, zu gründen. ${ }^{156}$ Ziel der Gründung einer eigenen Einrichtung war es, Lücken im Beratungs-, Infrastrukturund Raumangebot für Trans* - und Inter*-Menschen in Berlin zu schließen, indem die Einrichtung eine Anlaufstelle explizit und speziell für Trans*- und Inter*-Menschen schaffte. ${ }^{157}$ Anfangs nutzte TrIQ die Räume eines Ladenlokals, zog aber im Jahr 2011 in eine Fabrik-Etage um. Die ersten vier Jahre, also bis 2010, musste die Einrichtung ausschließlich mit ehrenamtlicher Arbeit auskommen. ${ }^{158}$ Mittlerweile verfügt die Einrichtung im Rahmen des »Inter* «- (2014) und des »Trans" Visible«-Projekts (2015) über jeweils zwei finanzierte Teilzeitstellen ${ }^{159}$ und zusätzlich eine Teilzeitstelle für Verwaltung, Koordination und Beratungsarbeit. ${ }^{160}$

Das Ziel von TrIQ ist, Räume für Trans*- und Inter*-Menschen zu schaffen, zu sensibilisieren und aufzuklären sowie die Interessen von Trans* - und Inter*-Personen

154 TransInterQueer e.V.: Publikationen, http://www.transinterqueer.org/unsere-publikationen/ vom 27.08.2020.

155 TransInterQueer e.V.: Über den Verein TransInterQueer, http://www.transinterqueer.org/ueber-triq /verein/ vom 27.08.2020.

156 Leo Yannick Wild: Mit Energie und unbändiger Lust, magazin.hiv 2016, https://magazin.hiv/2016/0 9/16/voller-energie-und-unbaendiger-lust/ vom 10.10.2020.

157 TransInterQueer e.V.: Warum dieses Projekt gebraucht wird, http://www.transinterqueer.org/uebe r-triq/warum-dieses-projekt-gebraucht-wird/ vom 27.08.2020.

158 L. Y. Wild: Mit Energie und unbändiger Lust.

159 Seit 2018 ist nur noch eine Person im »Inter*«-Projekt angestellt.

160 TransInterQueer e.V.: Wie funktioniert TrIQ?, http://www.transinterqueer.org/ueber-triq/wie-funkt ioniert-triq/ vom 27.08.2020. 
zu vertreten. Dabei wird der Anspruch, sich auf verschiedenen Wegen für ein diskriminierungsfreies und nicht-pathologisierendes Miteinander einzusetzen, ohne dabei den einen richtigen Weg vorgeben $\mathrm{zu}$ wollen, betont. ${ }^{161}$ Keine Selbstverständlichkeit in der queer-politischen Landschaft ist die Zusammenführung von Trans* - und Inter"-Interessen in ein gemeinsames Projekt. ${ }^{162}$ Gemeinsamer Nenner der Zusammenarbeit ist die Erfahrung, mit einer Zweigeschlechternorm konfrontiert zu sein, in der Trans* - und Inter*-Personen nicht vorgesehen sind. Vor allem der Kampf gegen Normierung und Pathologisierung und für ein Recht auf Selbstbestimmung über den eigenen Körper vermag in der Arbeit von TrIQ eine Brücke zwischen Trans* - und Inter* Interessen zu schlagen. Trans* und Inter* unter dem Dach einer gemeinsamen Einrichtung zu versammeln, bedeutet aber nicht nur gemeinsame Projekte, sondern auch Trans* - und Inter*-spezifische Projekte zu realisieren. ${ }^{163}$ Gefördert wird TrIQ seit 2010 von der Landesstelle für Gleichbehandlung - gegen Diskriminierung und dem Deutschen Paritätischen Wohlfahrtsverband Berlin. Im Rahmen des Programms »Demokratie leben!« bekommt TrIQ finanzielle Unterstützung vom Bundesministerium für Familie, Senioren, Frauen und Jugend. ${ }^{164}$ Die Arbeit von TrIQ setzt auf verschiedenen Ebenen an. Von TrIQ ausgehend werden kulturelle Austauschräume von und für Inter*- und Trans*-Personen, aber auch für Gruppentreffen organisiert und es finden regelmäßige Beratungen statt. Auch die beiden Einzel-Projekte schaffen bestimmte Räume des Austauschs und machen zudem Öffentlichkeits- und Bildungsarbeit. In der Öffentlichkeits- und Lobbyarbeit geht es vor allem darum, Gesellschaft, Politik, Medien und Mitarbeiter*innen aus dem Gesundheitsbereich zu erreichen. Hierbei spielen neben Broschüren, die der Sensibilisierung dienen, auch politische Stellungnahmen eine zentrale Rolle. In den politischen Stellungnahmen positioniert sich die Einrichtung zu aktuellen Trans* - und Inter*-relevanten Geschehnissen, wie beispielsweise die Bearbeitungen der Diagnosekriterien im ICD oder DSM. Zudem werden CommunityErinnerungstage genutzt, um Trans* - und Inter*-Themen einer breiten Öffentlichkeit sichtbar zu machen. ${ }^{165}$ Inhaltlich lässt sich die Arbeit von TransInterQueer e.V. in Form folgender Säulen darstellen:

a) Bildungs-, Öffentlichkeits- und Lobbyarbeit,

b) Beratung,

c) Kultur, Freizeit, Schutzräume.

161 Ebd.

162 Es gab und gibt vor allem auch aufgrund von Missverständnissen Meinungsverschiedenheiten und Konflikte zwischen Inter*- und Trans*-Vertretungen, wie Vereinnahmungen von Inter*- durch Trans*-Verbände oder ein Beharren von Inter* auf Biologismen, um sich von Trans* abzugrenzen.

163 TransInterQueer e.V.: Das »l« in TrlQ, http://www.transinterqueer.org/ueber-triq/das-i-in-triq/ vom 27.08.2020.

164 TransInterQueer e.V.: Unterstützer_innen, http://www.transinterqueer.org/ueber-triq/unterstuetze r_innen/vom 27.08.2020.

165 TransInterQueer e.V.: Öffentlichkeitsarbeit und Medien, http://www.transinterqueer.org/thema/of fentlichkeitsarbeit-medien/ vom 27.08.2020. 


\section{Beratung jenseits von Pathologisierung und Normierung}

Als Antwort auf die herkömmlichen medizinischen und psychologischen Beratungen, die Trans*- und Inter*-Menschen häufig pathologisieren und normative Körperlichkeiten und Geschlechtlichkeiten nicht hinterfragen, bietet TrIQ alternative Beratungskonzepte von und für Trans"- und Inter"-Personen an. In diesen Beratungen geht es explizit darum, Trans" - und Inter"-Menschen nicht - wie es in gängigen Beratungen häufig der Fall ist - einen richtigen Weg vorzugeben, sondern die Beratungen ergebnisoffen zu gestalten. Zentral ist, dass die Beratungsexpertise bei TrIQ nicht auf einer äußeren medizinischen Autorität fußt, sondern von Menschen kommt, für die Trans"- und Inter"-Erfahrungen zum eigenen Alltag gehören. Die Beratungen finden kostenlos und anonym statt. Es gibt die Möglichkeit der E-Mail-Beratung sowie - nach Terminvereinbarung - der telefonischen oder persönlichen Beratung. Einmal im Monat haben die Räume von TrIQ auch für Beratungen ohne Terminvereinbarungen geöffnet. Bei Bedarf kann die Beratung in Englisch, Französisch, Italienisch, Portugiesisch, Russisch, Spanisch, Tschechisch, Türkisch und Ungarisch stattfinden. Hierzu werden zumeist ehrenamtlich arbeitende Dolmetscher*innen hinzugezogen. Zielgruppe sind Trans"- und Inter"-Personen, aber auch Menschen, die beruflich mit dieser Zielgruppe zu tun haben. Bei TrIQ beraten Personen, die Expert*innen in eigener Sache sind. Beratungsthemen sind Geschlechtsidentität sowie rechtliche, medizinische, gesundheitliche und psychosoziale Fragen. Diese Beratung wird ergänzt durch vier weitere Beratungsformate. Zum einen gibt es eine spezifische Beratung für Trans"-Sexarbeiter"innen oder solche, die es werden wollen. Auch hier beraten aktive Trans"-Sexarbeiter"innen zu spezifischen Trans*-Gesundheitsthemen und zum spezifischen Arbeitsbereich an sich. Weitere Zusatzangebote sind die Trauma-Ambulanz und die systemische Lebensberatung. ${ }^{166} \mathrm{Zu}$ dem gibt es noch eine Sozialberatung, in der zu Themen wie Arbeitslosengeld 1 und 2, Arbeitsvermittlung im Jobcenter, Sozialgeld und Prozesskostenhilfe beraten wird. ${ }^{167} \mathrm{Da}$ Trans*- und Inter*-Menschen seltener einen Arbeitsplatz finden und häufiger Gewalt ausgesetzt sind, schließen die Beratungsangebote an diese spezifischen Trans"- und Inter"-Lebenswelten an. Auch Menschen, die nicht konkret Beratung suchen, können die nicht-pathologisierenden und -normierenden Austauschräume bei TrIQ nutzen.

\section{Kultur und Austausch}

Neben der Beratung werden von TrIQ ausgehend sichere Orte des Beisammenseins und des Austauschs geschaffen. Im Kunst- und Kulturbereich werden beispielsweise Workshops zu Tanzen, Musik, Schreiben, Diskutieren oder Comic zeichnen angeboten. Es gibt Slams, Konzerte und Performances. Es geht darum, Orte zu schaffen, an denen Trans*- und Inter"-Menschen jenseits von Stigmatisierung Zugang zu Kunst und Kultur bekommen. Über Medien wie Musik, Zeichnen und Schreiben sollen gemeinsam alternative Wirklichkeiten kennengelernt werden oder die eigenen Erfahrungen neu

166 TransInterQueer e.V.: Persönliche Trans*- und Inter*-Beratung, http://www.transinterqueer.org/be ratung/persoenliche-trans-inter-beratung/ vom 15.09.2020.

167 TransInterQueer e.V.: Sozialberatung, https://www.transinterqueer.org/beratung/sozialberatung/ vom 15.09.2020. 
gedeutet werden. Kunst- und Kulturveranstaltungen bieten zudem eine niederschwellige Möglichkeit des Beisammenseins oder des gemeinsamen Austauschs. ${ }^{168}$ Jenseits des Kunst- und Kulturbereichs finden bei TrIQ Workshops statt, in denen verschiedene Fertigkeiten weitergegeben oder bestimmte Themen diskutiert werden. So wurden beispielsweise Workshops zum Verschlüsseln von E-Mails und zum Job-Mentoring durchgeführt. In anderen Workshops ging es wiederum darum, sich zu Trans* und Elternschaft oder zum Inter*-Sprachgebrauch auszutauschen. Je nach Thema werden diese zum Teil nur für bestimmte Zielgruppen geöffnet. ${ }^{169}$ Neben diesen zumeist unregelmäßigen Veranstaltungen finden unter dem Dach von TrIQ auch regelmäßige Treffen verschiedener Gruppen statt, meistens direkt in den Räumen von TrIQ. Zumeist leiten und koordinieren ein bis zwei Personen die Gruppen. 2019 wurden auf der Homepage 16 Gruppen aufgelistet, von denen aber einige aus unterschiedlichen Gründen inzwischen stillgelegt sind. ${ }^{170}$ Es gibt Gruppen, in denen gemeinsam Sport und Yoga gemacht oder meditiert wird. In anderen Gruppen stehen bestimmte Themen im Vordergrund, wie zum Beispiel in der geschlechtskritischen »Passing «-Gruppe. ${ }^{171}$ Andere Gruppen organisieren sich um eine bestimmte Zielgruppe, wie zum Beispiel »Trans*-Personen im Alter«, »Trans*-Männer und Genderboys« oder »weder* noch*-Menschen«. Dabei wird in den Gruppen versucht, von Beginn an Räume herzustellen, in denen eben keine normative Vorstellung von dem, was Trans* oder Inter* bedeutet, existiert. ${ }^{172}$ Neben der Beratung und den gemeinsamen Räumen des Beisammenseins und des Austauschs gibt es bei TrIQ zwei finanzierte Projekte: das »Inter*«-Projekt und das »Trans* Visible«Projekt.

\section{Das »Inter* «-Projekt}

Das Projekt »Antidiskriminierungsarbeit \& Empowerment für Inter" « (kurz »Inter" «Projekt) wurde 2014 gestartet. Gefördert wird es von der Berliner Senatsverwaltung für Arbeit, Integration und Frauen, der »Initiative Berlin tritt ein für Selbstbestimmung und Akzeptanz sexueller Vielfalt « und der Landesstelle für Gleichbehandlung gegen Diskriminierung. Das Projekt beziehungsweise das Projektkonzept gründet auf einer wissenschaftlichen Bedarfserhebung. Ausgehend von sich überschneidenden Alltagserfahrungen, die von vielen Inter*-Personen geteilt werden, konnten so vier Säulen der Arbeit für das Projekt herausgefiltert werden: a) Sprache, b) Gemeinschaft, c) Aufklärung der Gesellschaft, d) Antidiskriminierungsarbeit in strukturellen Räumen.

Die Öffentlichkeitsarbeit war zu Beginn zentral für das »Inter*«-Projekt. Neben zum Teil sehr umfangreichen Stellungnahmen und Pressemitteilungen entstanden seit 2014 zwei große Broschüren. Zum Thema »Sprache« wurde eine eigene Broschüre -

168 TransInterQueer e.V.: Kunst \& Kultur, http://www.transinterqueer.org/thema/kunst-kultur/ vom 27.08.2020.

169 TransInterQueer e.V.: Fortbildungen \& Workshops, https://www.transinterqueer.org/thema/fortbil dung/ vom 27.08.2020.

170 Bei einer Gruppe gibt es zu wenige Teilnehmer*innen, bei anderen Gruppen steht, dass die Gruppe während der momentanen Raumsuche pausiert.

171 Hier unterstützen sich Menschen gegenseitig dabei, die Ceschlechtsidentität »besser« zu performen, die der jeweiligen Identität entspricht.

172 TransInterQueer e.V.: Gruppen, https://www.transinterqueer.org/gruppen/vom 27.08.2020. 
»Inter* und Sprache ${ }^{173}$ - erstellt, in der positive Arten des Sprechens über Inter* bestärkt werden und über negative, diskriminierende Sprache aufgeklärt wird. Eine zweite Broschüre, die im Rahmen des »Inter* «-Projekts erstellt wurde, ist die Broschüre "Medizinische Eingriffe an Inter* und deren Folgen: Fakten und Erfahrungen ${ }^{174}$, in der Inter*-Menschen über die an ihnen vorgenommenen Eingriffe berichten. Hier geht es darum, die Gesellschaft generell für die oft gewaltdurchzogenen Lebenswelten von Inter*-Personen zu sensibilisieren, also aufzuklären. Auf der Ebene der Gemeinschaftsbildung wurde im Zuge des »Inter"«-Projektes eine Kunstwerkstatt ins Leben gerufen, in der gemeinsam kreativ gemalt und gebastelt wird und die primär dem Empowerment von Inter*-Menschen dient. ${ }^{175}$ Während zu Beginn des »Inter* «-Projekts die Öffentlichkeits- und Lobbyarbeit im Vordergrund stand und entsprechend umfangreiche Broschüren, aber auch Stellungnahmen veröffentlicht wurden, spielt mittlerweile vor allem die Gemeinschaftsbildung eine zentrale Rolle. ${ }^{176}$

\section{Das "Trans* Visible«-Projekt}

»Trans* Visible - Wissen und Support für Akzeptanz - gegen Gewalt« (kurz: »Trans* Visible«) hat zum Ziel, auf unterschiedlichsten Ebenen die Sichtbarkeit von Trans*Menschen zu erhöhen. ${ }^{177}$ Das Projekt wird vom Bundesministerium für Familie, Senioren, Frauen und Jugend im Rahmen des Programms "Demokratieleben! « gefördert. ${ }^{178}$ »Trans" Visible« ist ein Modellprojekt, das im Jahr 2015 gestartet hat und auf fünf Jahre angelegt ist. ${ }^{179}$ "Trans* Visible« besteht aus mehreren kleineren Projekten und Kampagnen. Nachdem Medien für die Art und Weise, wie Trans* sichtbar wird, eine zentrale Rolle spielen, kommt der medialen Darstellung von Trans* eine wichtige Rolle im »Trans* Visible«-Projekt zu. So organisierte beispielsweise die Medienkampagne »Gender Sender« Workshops, in denen filmisch eine kreative und kritische Auseinandersetzung mit Geschlecht umgesetzt wurde. Diese Filme sollten dann wiederum für Sensibilisierungs- und Fortbildungsworkshops verwendet werden. Ebenfalls mit Bezug auf die Repräsentation von Trans* in den Medien werden im Zuge des Unterprojekts »Medienwatchdog« externe journalistische Print-, Online-, Radio- und TV-Produkte

173 TransInterQueer e.V.: Inter* und Sprache. Von >Angeboren< bis >Zwitter< 2015, https://www.transint erqueer.org/download/Publikationen/InterUndSprache_A_Z.pdf vom 15.09.2020.

174 TransInterQueer e.V.: Medizinische Eingriffe an Inter* und deren Folgen: Fakten \& Erfahrungen 2016, https://www.transinterqueer.org/download/Publikationen/eingriffe_broschuere_inter_2016_ sm.pdf vom 15.09.2020.

175 TransInterQueer e.V.: Inter*Projekt, https://interprojekt.wordpress.com/ vom 15.09.2020.

176 Diese Informationen stammen aus dem Interview.

177 TransInterQueer e.V.: Begleitforschung bei Trans* Visible, http://www.transinterqueer.org/wp-cont ent/uploads/Begleitforschung.pdf vom 06.09.2020.

178 Weitere Unterstützer*innen sind die Bundesstiftung Magnus Hirschfeld, die HannchenMehrzweck-Stiftung, die Homosexuelle Selbsthilfe e.V., Interflugs, Naturfreundejugend, Stiftung DKLB und DPW, LV Berlin*, Schwulenberatung Berlin CmbH, Schwuz, Spender_innen und Wigstöckel e.V. Des Weiteren findet auch eine Zusammenarbeit mit dem Gunda-Werner-Institut der Heinrich-Böll-Stiftung statt.

179 Die weitere Förderung wird allerdings nur unter der Voraussetzung garantiert, dass TriQ selbst auch finanzielle Eigenmittel für das Projekt beschafft. 
zum Thema Trans* in einer Datenbank gesammelt. ${ }^{180}$ Neben dieser Online-Datenbank bietet auch das "Trans*Inter*-Archiv« die Möglichkeit, in den Räumen von TrIQ zu Trans* - und Inter*-Themen zu recherchieren. Hier finden sich Fachliteratur, Belletristik und Zeitschriftensammlungen rund um Trans* - und Inter*-Themen. Es werden aber auch Möglichkeiten bereitgestellt, selbst Öffentlichkeiten herzustellen. Hierzu werden Podcasts, als Medien, die leicht produzierbar und publizierbar sind, genutzt. Auf der Medienplattform »ti-Blogs«, die im Zuge von »Trans* Visible« errichtet wurde, können Trans* - und Inter*-Menschen ihre Podcasts veröffentlichen. ${ }^{181}$ Das $»$ Trans* Visible«Projekt arbeitet neben dem sehr starken Medienfokus auch zu den Themen »Trans* und Sexarbeit«, »Trans* Inter* Queer im Alter« und »Trans*-Gewalterfahrungen«. Es gibt ein regelmäßiges Treffen von und für Trans*-Sexarbeiter*innen. Es wird der Beratungsbedarf von Sexarbeiter*innen ermittelt und eine mehrsprachige Broschüre für Trans*-Sexarbeiter*innen erstellt. Für »Trans* Inter* Queer im Alter « wird ein Besuchsprojekt konzeptualisiert und aufgebaut, es gibt Vernetzungen mit Multiplikator*innen und Berichterstattung zum Thema. Auch das Thema »Gewalt« findet sich in diesem Projekt. So werden beispielsweise seit 2016 die Datenbanken zu Hate Crime in Deutschland ausgebaut. Darüber hinaus spielt generell die Lobbyarbeit eine wichtige Rolle im »Trans* Visible«-Projekt. Es geht darum, Wissen zu rechtlichen, medizinischen und anderen Trans*-Themen, in Form von Vorträgen oder Tagungen, zu vermitteln und Bedürfnisse und Forderungen an politische Entscheidungsträger*innen heranzutragen. $^{182}$

Neben den zwei gut finanzierten Projekten gibt es bei TrIQ mittlerweile auch ein »Refugee@TrIQ «-Projekt, ${ }^{183}$ in dem geflüchtete Trans*-Personen beraten, informiert und empowert werden sowie Öffentlichkeits- und Bildungsarbeit zum Thema geleistet wird. Zudem gibt es ein eigenes Radio, das »TransgenderRadio«, ${ }^{184}$ und mittlerwei-

180 Im Zuge dieses Projektes werden auch Schulungen zur Nutzung und Recherche in den Datenbanken, Workshops und Fortbildungen für Journalist*innen sowie Vernetzung mit - mit Trans*Themen vertrauten - Medienanwält*innen angeboten.

181 Ein etwas kleineres und nicht finanziertes Projekt, das auch auf der medialen Ebene agiert, um eine alternative Öffentlichkeit herzustellen, ist das »TransgenderRadio«, das es seit 2011 gibt. Hier produzieren Menschen für die »Ceschlecht - entgegen dem scheinbar so sicheren Alltagswissen keine natürliche Kategorie ist, sondern auf Vereinbarungen, Zwängen, Konstruktionen und Spiel beruht«, einmal im Monat eine Radiosendung. Zurzeit läuft die Sendung samstags zwischen 11 und 12 Uhr und wird in Berlin, Dresden, (Zürich), Wien, Graz, Leipzig und Halle ausgestrahlt. In der Radiosendung gibt es Interviews, aktuelle Trans-* und Inter*-Themen werden vorgestellt und diskutiert und prominente Trans*-Personen eingeladen, es gibt Kultur- und Veranstaltungstipps und Musik wird gespielt. Ziel ist es, Zweigeschlechtlichkeiten »auf den Prüfstand «zu stellen und über aktuelle Gesetzeslagen und politische Entscheidungen zu informieren. In den Räumen von TrIQ kann bei einem Frühstück auch gemeinsam die Radiosendung angehört werden.

182 TransInterQueer e.V.: Trans* Visible, https://www.transinterqueer.org/projekte/trans-visible/ vom 15.09.2020.

183 TransInterQueer e.V.: Refugees@TrlQ, https://www.transinterqueer.org/refugees/ vom 27.08.2020.

184 TransInterQueer e.V.: Transgenderradio, https://www.transinterqueer.org/projekte/transgenderrad io/ vom 27.08.2020. 
144 Das politische Subjekt des queeren Aktivismus

le ist die Beratungs- und Bildungsarbeit unter anderem speziell auf Vornamens- und Personenstandsänderungen ausgelegt. ${ }^{185}$

185 TransInterQueer e.V.: Personenstand, https://www.transinterqueer.org/pstg/ vom 27.08.2020. 